\title{
Ice condition assessment using onboard accelerometers and statistical change detection
}

\author{
Heyn, Hans-Martin ; Blanke, Mogens; Skjetne, Roger
}

Published in:

I E E E Journal of Oceanic Engineering

Link to article, DOI:

10.1109/JOE.2019.2899473

Publication date:

2020

Document Version

Peer reviewed version

Link back to DTU Orbit

Citation (APA):

Heyn, H-M., Blanke, M., \& Skjetne, R. (2020). Ice condition assessment using onboard accelerometers and statistical change detection. I E E E Journal of Oceanic Engineering, 45(3), 898-914.

https://doi.org/10.1109/JOE.2019.2899473

\section{General rights}

Copyright and moral rights for the publications made accessible in the public portal are retained by the authors and/or other copyright owners and it is a condition of accessing publications that users recognise and abide by the legal requirements associated with these rights.

- Users may download and print one copy of any publication from the public portal for the purpose of private study or research.

- You may not further distribute the material or use it for any profit-making activity or commercial gain

- You may freely distribute the URL identifying the publication in the public portal 


\title{
Ice condition assessment using onboard accelerometers and statistical change detection
}

\author{
Hans-Martin Heyn, Member, IEEE, Mogens Blanke, SM, IEEE, Roger Skjetne, SM, IEEE,
}

\begin{abstract}
The presence of sea ice is the predominant risk for ship operations in the Arctic, and monitoring of ice condition around a vessel is crucial during all times of operation. This paper presents a system for online onboard assessment of ice condition. It is demonstrated that ice-induced accelerations in the bow section of the hull follow a bivariate t-distribution and that parameters of the distribution have a one to one relation to ice condition. The paper suggests a methodology to monitor the ice condition in real time through estimation of parameters that characterise the distribution of hull accelerations. It is shown how a Kullback-Leibler divergence measure can classify ice condition among a set of pre-trained conditions. An absolute measure of ice load is suggested as an alternative for situations when pre-training data are not available. The alternative algorithm quantifies the condition through the entropy of measured accelerations. The article presents a computationally easy methodology and tests against data collected during Arctic transit of an icebreaker. Further, the classification results are compared with the results from two standard methods from machine learning, decision tree and a support vector machine approaches. The results show that the statistical methods provide robust assessment of the prevailing ice conditions, independent of visual and weather conditions. The also comparison shows that the statistical classification methods, designed by process knowledge, provide steadier and more reliable results.
\end{abstract}

Keywords-Arctic; bivariate t-distribution; entropy; generalised log-likelihood ratio; IMU; Kullback-Leibler divergence; machine learning; ocean engineering; sea ice; ship-ice interaction, statistical change detection.

\section{INTRODUCTION}

Operations in the Arctic are heavily influenced by ice loads as the predominant risk factor. Accidents impairing the fragile environment of the Arctic have to be avoided at any cost [1]. Therefore, ice management ensures that ice loads on a protected vessel do not exceed its structural and operational safety limits [2]. Ice management relies on ice intelligence, which is the process of collecting and processing information about the ice environment [3], [4]. It is beneficial to collect this information in situ, without the need of external platforms such as satellites, drones, or buoys. The system collecting the information should be able to operate without weather-related

Hans-Martin Heyn is with the Department of Marine Technology, Norwegian University of Science and Technology, Trondheim, Norway e-mail: martin.heyn@ntnu.no

Mogens Blanke is with the Automation and Control Group, Department of Electrical Engineering, Technical University of Denmark, Kgs. Lyngby, Denmark e-mail: mb@elektro.dtu.dk

Roger Skjetne is with the Department of Marine Technology, Norwegian University of Science and Technology, Trondheim, Norway

Manuscript submitted January 06, 2019;

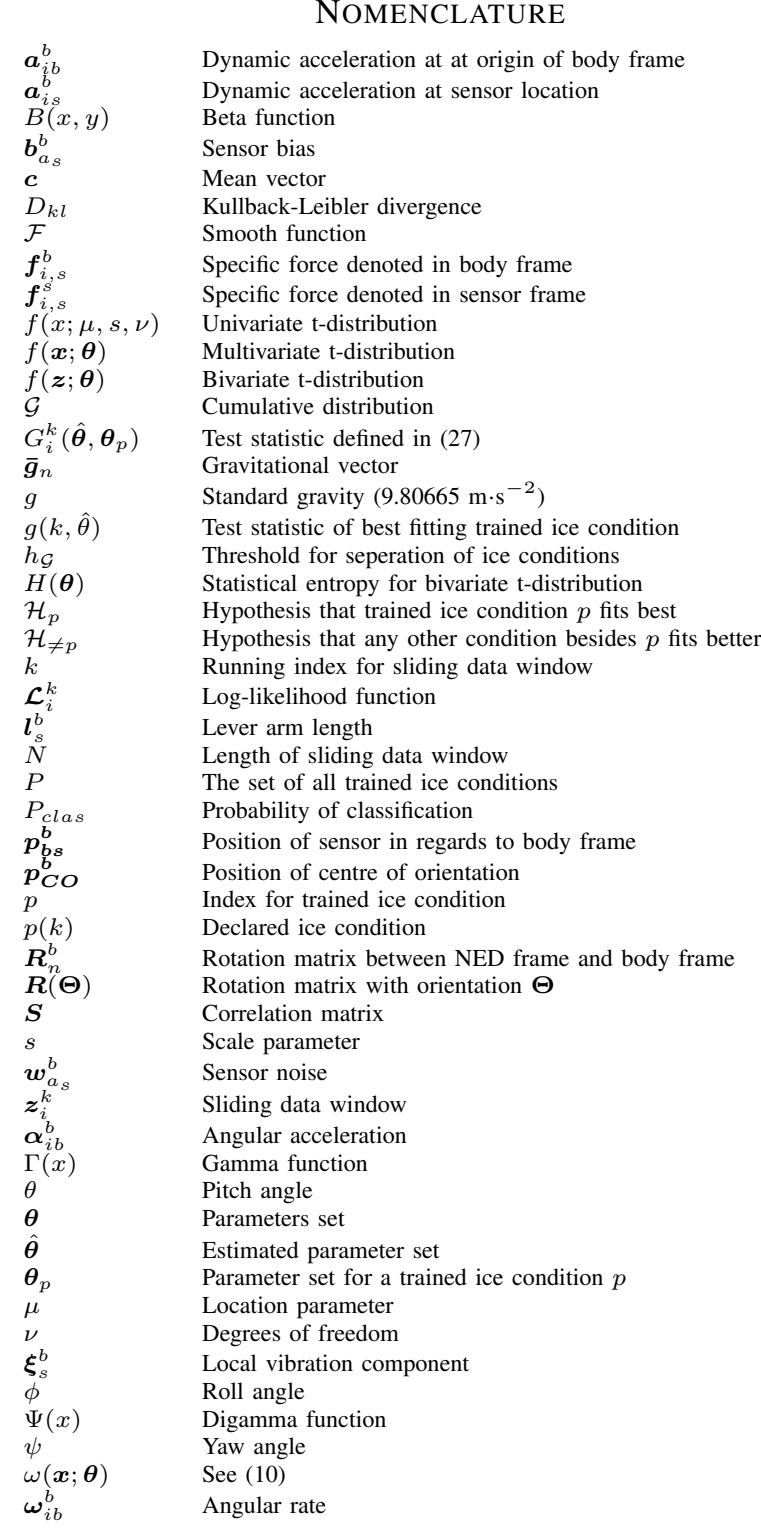


limitations. It should be possible to process ice information in real-time into a format, which is useful as decision support for operators, and which can be used as input to a control and monitoring system.

Present ship-based ice observation systems employ optical cameras, e.g. [5], [6], and image processing extracts information such as ice concentration, floe size distribution [7]-[11], and ice thickness [12]. Camera based ice intelligence becomes unavailable in harsh weather conditions, such as fog or heavy snow. Shipborne synthetic aperture radar (SAR) systems can help to identify ice fields, but SAR is unreliable in the close vicinity of the vessel [13]-[15]. Inertial measurement units (IMUs) provide direct information about exterior loads on the vessel. IMUs are inexpensive, easy to install, and do not depend on weather conditions. Johnston et al. [16] showed that shipboard IMUs provide global loads from interaction with sea ice. Kjerstad et al. [17], [18] extended the approach to four distributed IMUs. These approaches provided global load information. It is desired to obtain spatial information about the surrounding ice, taking into account that the ship's hull is not a rigid, but rather a flexible and deformable body. Strain gauges measure strain and bending. Locally induced ice loads can be extracted from such measurements [19], [20]. In contrast to IMUs, strain gauges are time-consuming to install, and thus expensive [21]. This study therefore focus on the problem of assessing local ice condition based on measurements by IMUs placed in the hull of the ship.

Changes in ice load depend on the vessel's velocity, shape of the hull, and on the properties of the ice, such as density, thickness, and salinity [22], [23]. The properties of the ice have a large local variability, which results in sudden changes in magnitude of the ice-induced loads, especially upon the encounter of ice ridges [24]. Studies conducted with data from strain gauges suggested that the measured ice loads could be described statistically [25], e.g., by a Weibull distribution [26], by a log-normal distribution [19], or by a 3-parameter exponential distribution [27]. The Weibull and log-normal distributions are heavy tailed distributions. A recent study [28] showed that local ice-induced vibrations, measured by IMUs in the hull of an icebreaker, follow a non-central, scaled tdistribution, where the shape changes with the ice condition.

The goal of the paper is to develop and validate a fast, online, shipborne ice assessment system based on the estimation of parameters in the distribution of local accelerations. Data from in-plane accelerometers are used to find a statistical model of local ice-induced accelerations. Typical distribution parameters for four ice conditions are determined using training data. Under operations, the distribution parameters are estimated for small time windows and compared to the parameter sets from the trained ice conditions. The paper proposes two custom methods to assess ice-induced hull accelerations: Method one employs a modified KullbackLeibler divergence to find the trained ice condition that best describes the measured data. Method two utilises directly the change in signal entropy to classify the severity of ice loads. The results from both classification approaches are validated using data obtained during the transit of an icebreaker. Data from a camera system that observed the visual ice condition is
TABLE I: Technical data of icebreaker Oden [31].

\begin{aligned} & \hline Length $107.75 \mathrm{~m} \\ &$ Beam $31.2 \mathrm{~m} \\ &$ Draft $7.0-8.5 \mathrm{~m} \\ &$ Total power $18 \mathrm{MW} \\ &$ Speed in open water $15 \mathrm{knots} \\ &$ Icebreaking capability $1.9 \mathrm{~m} \mathrm{level} \mathrm{ice} \mathrm{at} 3$ knots \\ & Bunker capacity $4600 \mathrm{~m}^{3} \\ &$ Displacement $11.000-13.000 \mathrm{t} \\ &$ Propulsion 4 medium speed diesel engines \\ & 2 propellers in nozzles \\ & \hline\end{aligned}

used for reference. Furthermore, the classification results are compared with the results from two trained machine learning classification models, namely a trained decision tree model and a trained support vector machine (SVM) model.

The paper is structured as follows. Section II introduces the experiment setup and documents training data for different ice conditions. Section III describes how ice-induced accelerations are modelled by a bivariate t-distribution and derives a maximum-likelihood estimator for recursive parameter estimation of the distribution. Section IV presents the statistical methods for ice condition classification seconded by two standard machine learning approaches. Section V validates the classification approaches with data sets obtained onboard an icebreaker. Furthermore, the section also presents a comparison between the custom classification approaches and the two trained machine learning models. Section VI assess the robustness of results and suggests how the monitoring technology could best be applied. A final section provides discussion of results and conclusions.

\section{PROBLEM FORMULATION}

The problem at hand is to determine the ice condition and to capture changes in ice condition at a section of the ship's hull from local acceleration measurements in the interior structure. The detection of a change in ice condition must be fast enough to detect sudden changes in ice thickness, which typically occur when the ship encounters an ice ridge [19], [29]. The system must therefore detect changes within seconds to give enough time for the crew or a control system to prevent getting locked-in ice.

\section{A. Measurement setup}

A test system was established with two IMUs installed on the Swedish icebreaker Oden during the Arctic Ocean 2016 expedition [30]. A summary of the icebreaker's technical specifications is given in Table I.

Figure 1 illustrates the placement of sensors. IMU 1 was installed close to the geometrical centre of symmetry of the hull. Its purpose was to provide reference data of the global accelerations acting on the vessel. IMU 2 was installed inside the hull, at the ice knife of the vessel. This unit recorded local vibrations in the bow section of the hull. The data provided by IMU 2 will be used for the ice diagnostic system, with the aim to detect changes in ice condition at the bow section. The full technical specifications of the IMUs can be found in [32]. 


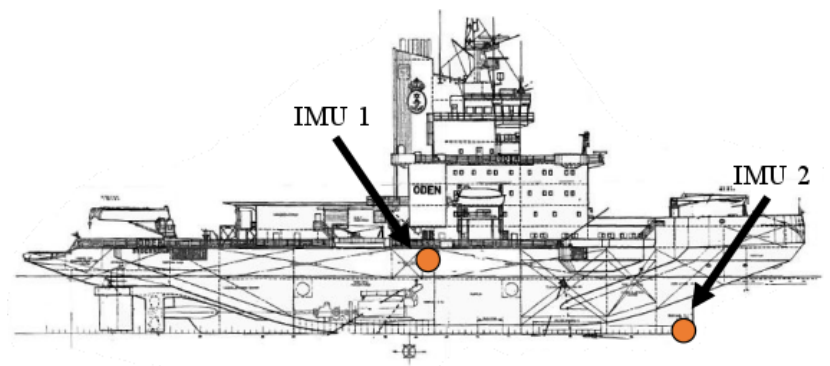

Fig. 1: Placement of the inertial measurement units (IMUs).

\section{B. Model of the sensor data}

Each sensor contains three orthogonal accelerometers as illustrated in Figure 2. The three accelerometers record the specific forces, $\boldsymbol{f}_{i s}^{s} \in \mathbb{R}^{3}$ in the sensor frame $\{s\}$ with respect to an inertial frame $\{i\}$. A rotation matrix $\boldsymbol{R}\left(\boldsymbol{\Theta}_{b s}\right)$ (see [33], Eq. 2.18), transforms the recorded specific forces to the ship's body frame $\{b\}$, where $\boldsymbol{\Theta}_{b s}=\left(\phi_{s}, \theta_{s}, \psi_{s}\right)$ describes the orientation of the sensor, see Table II:

$$
\boldsymbol{f}_{i s}^{b}=\boldsymbol{R}\left(\boldsymbol{\Theta}_{b s}\right) \boldsymbol{f}_{i s}^{s} .
$$

The rotated sensor output for the accelerations is modelled in standard literature, [33], [34]:

$$
\boldsymbol{f}_{i, s}^{b}=\boldsymbol{a}_{i s}^{b}-\boldsymbol{R}_{n}^{b} \overline{\boldsymbol{g}}^{n}+\boldsymbol{b}_{a_{s}}^{b}+\boldsymbol{w}_{a_{s}}^{b},
$$

where $\boldsymbol{a}_{i s}^{b} \in \mathbb{R}^{3}$ is the dynamic acceleration in the location $s$ of the sensor with respect to $\{i\}, \boldsymbol{R}_{n}^{b} \in \mathbb{R}^{3 \times 3}$ is the rotation matrix between the north-east-down (NED) frame $\{n\}$ and $\{b\}, \overline{\boldsymbol{g}}^{n}=(0,0, g)$ is the gravitational vector [34] in $\{n\}$, $\boldsymbol{b}_{a_{s}}^{b} \in \mathbb{R}^{3}$ contains the sensor bias, and $\boldsymbol{w}_{a_{s}}^{b} \in \mathbb{R}^{3}$ accounts for the sensor noise. The translational accelerations $\boldsymbol{a}_{i s}^{b}$ can be transferred to the origin $b$ of the ship's body frame (i.e., the centre of orientation $(C O)$ ),

$$
\boldsymbol{a}_{i b}^{b}=\boldsymbol{a}_{i s}^{b}-\boldsymbol{S}\left(\boldsymbol{\omega}_{i b}^{b}\right)^{2} \boldsymbol{l}_{s}^{b}-\boldsymbol{S}\left(\boldsymbol{\alpha}_{i b}^{b}\right) \boldsymbol{l}_{s}^{b},
$$

where $\boldsymbol{\omega}_{i b}^{b} \in \mathbb{R}^{3}$ and $\boldsymbol{\alpha}_{i b}^{b}=\dot{\boldsymbol{\omega}}_{i b}^{b}$ are the angular velocities and accelerations of $b$ relative to $\{i\}$, respectively, and $\boldsymbol{l}_{s}^{b}=$ $\boldsymbol{p}_{b s}^{b}-\boldsymbol{p}_{\mathrm{CO}}^{b}$ is the lever arm from $\{b\}$ to $\{s\} . \boldsymbol{S}(\boldsymbol{\omega})$ is the skewsymmetric matrix, that represents the cross product operator $\boldsymbol{\omega} \times$,

$$
\boldsymbol{S}(\boldsymbol{\omega})=\left[\begin{array}{ccc}
0 & -\omega_{z} & \omega_{y} \\
\omega_{z} & 0 & -\omega_{x} \\
-\omega_{y} & \omega_{x} & 0
\end{array}\right]
$$

The measured accelerations $\boldsymbol{f}_{i, s}^{b}$ at the sensor's location contain a translational component $\boldsymbol{a}_{i s}^{b}$, and in addition a local vibration component $\boldsymbol{\xi}_{s}^{b}$, which is typically damped out through the flexibility of the hull structure and attenuated in the vessel's origin $b$. Hence, an IMU located in $C O$ may not feel the local vibration component at the bow; however, when transforming the measurements $f_{i, s}^{b}$ in $s$ to $C O$, the vibrations are part of
TABLE II: Placement of IMUs relative to $C O$.

\begin{tabular}{rr|rr}
\hline & & IMU 1 & IMU 2 \\
\hline \multirow{3}{*}{$\boldsymbol{l}_{s}^{b}$} & $x$ & $0 \mathrm{~m}$ & $33.95 \mathrm{~m}$ \\
& $y$ & $0 \mathrm{~m}$ & $0 \mathrm{~m}$ \\
& $z$ & $0 \mathrm{~m}$ & $-8.39 \mathrm{~m}$ \\
\hline \multirow{3}{*}{$\Theta_{b s}$} & $\phi_{s}$ & $-0.58^{\circ}$ & $-2.90^{\circ}$ \\
& $\theta_{s}$ & $180.45^{\circ}$ & $180.05^{\circ}$ \\
& $\psi_{s}$ & $106.00^{\circ}$ & $90.00^{\circ}$ \\
\hline
\end{tabular}

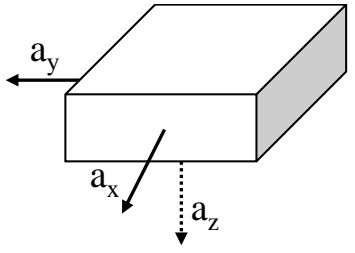

Fig. 2: IMU sensor containing three accelerometers.

the signal, according to

$$
\begin{aligned}
\boldsymbol{a}_{i b}^{b}= & \tilde{\boldsymbol{a}}_{i s}^{b}-\boldsymbol{S}\left(\boldsymbol{\omega}_{i b}^{b}\right)^{2} \boldsymbol{l}_{s}^{b}-\boldsymbol{S}\left(\boldsymbol{\alpha}_{i b}^{b}\right) \boldsymbol{l}_{s}^{b} \\
= & \boldsymbol{f}_{i, s}^{b}+\boldsymbol{R}_{n}^{b} \overline{\boldsymbol{g}}^{n}-\boldsymbol{S}\left(\boldsymbol{\omega}_{i b}^{b}\right)^{2} \boldsymbol{l}_{s}^{b}-\boldsymbol{S}\left(\boldsymbol{\alpha}_{i b}^{b}\right) \boldsymbol{l}_{s}^{b} \\
& -\boldsymbol{b}_{a_{s}}^{b}-\boldsymbol{\xi}_{s}^{b}-\boldsymbol{w}_{a_{s}}^{b},
\end{aligned}
$$

with $\tilde{\boldsymbol{a}}_{i s}^{b}=\boldsymbol{a}_{i s}^{b}-\boldsymbol{\xi}_{s}^{b}$ being the vibration free acceleration at the sensor's location. The rotated in-plane specific forces $f_{i s, x}^{b}$ and $f_{i s, y}^{b}$ were utilised in this study.

\section{Mapping of roll- and pitch angles from acceleration mea- surements}

Under operations, the ship experiences roll and pitch motions, which change the attitude angle of the ship. Under the assumptions that the average acceleration with respect to the environment is zero for a sufficiently large data window [33] and the accelerometers are calibrated to remove bias, the total additional roll and pitch angles experienced by the sensors can be estimated by:

$$
\begin{aligned}
\phi_{a d d} & \approx \operatorname{atan} 2\left(-\bar{a}_{y},-\bar{a}_{z}\right) \\
\theta_{a d d} & \approx-\operatorname{atan} 2\left(\bar{a}_{x}, \sqrt{\bar{a}_{y}^{2}+\bar{a}_{z}^{2}}\right),
\end{aligned}
$$

where $\operatorname{atan} 2(y, x)$ provides an angle in the interval $(-\pi, \pi]$. If gyro data were available, they could be utilised to enhance the precision of the attitude estimation, using an extended Kalman filter or nonlinear observers; see [35].

\section{Preprocessing}

Preprocessing of accelerometer data were performed as follows:

- To assure that sensor noise is not entering the diagnostic system, and to accelerate processing, the data recorded at $100 \mathrm{~Hz}$, were decimated to $20 \mathrm{~Hz}$, with prior lowpass filtering to $10 \mathrm{~Hz}$ and subsequent downsampling. It was shown in [36], [37] that a frequency spectrum up to $10 \mathrm{~Hz}$ allows for the capture of relevant ice-induced hull vibrations. The utilised lowpass filter was a Chebyshev Type I filter of order 8 with a passband ripple of $0.05 \mathrm{~dB}$.

- Each accelerometer was calibrated to minimise the measurement bias. 
- Influence of roll and pitch were removed in preprocessing using (6) and (7) on the mean of a sufficiently large data window.

- The in-plane (in body frame coordinates) acceleration measurements were used. The horizontal plane acceleration will be shown to follow a bivariate statistical distribution, see III-B. This distribution has clear mathematical advantages. By omitting the vertical acceleration, compensation of fast pitch motions was not needed.

\section{E. Training data sets}

Training data sets were recorded under the following conditions:

- The training data sets were collected at a constant speed of the vessel ( 5 knots). The result of the ice condition assessment depends on the speed of the vessel. Therefore the validation data were recorded under normal icebreaking velocities of the vessel (3-7.5 knots).

- Because the sensor was placed in the bow, the ice action had to occur against the bow of the vessel.

Four ice conditions are shown in Figure 3. The upper left image shows the condition of open water, denoted as $\mathcal{H}_{O W}$. The upper right image shows a condition of open ice, $\mathcal{H}_{O I}$. The lower left image shows close ice, $\mathcal{H}_{C I}$, and the lower right image shows very close ice, $\mathcal{H}_{V C I}$. Half a minute of accelerations were recorded by the IMUs under each ice condition. These data were used as training data for the detection system. The rotated $\mathrm{x}$ - and $\mathrm{y}$-acceleration data from IMU 1 and IMU 2 are depicted in Figures $4 \mathrm{a}$ and 4b, respectively, for each of the ice conditions. The scatter plots also include the $99 \%$-confidence ellipses for data from each ice condition. The measurements from both IMUs, for all ice conditions, are centred near the origin. Furthermore, the radius of the confidence ellipses increases with the severity of the ice conditions. The reason is that the measured accelerations scatter significantly more under heavy ice conditions due to an increase in variance of the recorded signals. The data from IMU 2 show significantly more scattering for each ice condition than data from IMU 1. The additional local vibrations $\boldsymbol{\xi}_{s}^{b}$, present at the location of IMU 2, are assumed to give rise to this behaviour. Therefore, the measurements from the in-plane accelerometers of IMU 2 will be used throughout this study. Measurements from IMU 1 were discarded because they do not contain local ice-induced hull vibrations that would allow for a clear distinction of ice conditions based on acceleration data.

\section{F. Problem statement}

The change in ice conditions around a vessel is a continuous process. The severity of ship-ice interaction can be classified into a set of conditions, each of which characterise the distribution of hull accelerations. Table III describes four trained ice conditions. The number of ice conditions is arbitrary and can be increased if a more fine-grained classification is of interest. The ice condition can change without notice at an unknown change time $k_{0}$. The $\mathrm{x}$ - and $\mathrm{y}$-components of the
TABLE III: Trained ice conditions.

\begin{tabular}{cll}
\hline Hypothesis & Ice condition & Ice cover* \\
\hline $\mathcal{H}_{\mathrm{OW}}$ & Open water & $<10 \%$ \\
$\mathcal{H}_{\mathrm{OI}}$ & Open pack ice & $11 \%-60 \%$ \\
$\mathcal{H}_{\mathrm{CI}}$ & Close pack ice & $61 \%-80 \%$ \\
$\mathcal{H}_{\mathrm{VCI}}$ & Very close pack ice & $81 \%-100 \%$ \\
\hline *Visual ice coverage on sea surface. &
\end{tabular}

acceleration vector described in (5) are congregated in $\boldsymbol{z}$. At each time instance $k$, the last $N$ measurements are considered: $z \in \mathbb{R}^{2 \times N}$. The measurements are assumed independent and distributed with a bivariate probability distribution $f(\boldsymbol{z} ; \boldsymbol{\theta})$. To show that statistical independence can be justified, the appendix provides a correlogram of the measurement data from case 1 (see Section V.A). The parameters $\boldsymbol{\theta}$ of this joint probability distribution change with the ice condition. Following the concept of change detection, described in [38], at a time instant $k$ it must be decided which of the trained ice conditions fits the recorded data best. The problem consists of three parts:

- Estimation of the current parameter vector $\boldsymbol{\theta}$.

- Generation of a test statistic.

- Decide on an ice condition hypothesis $\mathcal{H}_{p}$.

This combination of estimation of parameters from given signals, change detection from an estimated set of parameters, and declaring a hypothesis from a test statistic defines a diagnostic system, as shown in Figure 5. The hypothesis testing is in its principles similar to the one-vs-all multi-class extension for support vector machines (SVMs) [39]. For each condition a hypothesis test exists to distinguish the data sample between two hypotheses, namely accept the condition or accept another condition.

\section{PARAMETER ESTIMATION IN BIVARIATE T-DISTRIBUTED DATA}

\section{A. Statistical change detection for heavy-tailed data}

Hypothesis testing in Gaussian distributed data is a well established field of research, and an extensive overview can be found in [40]. Detecting changes in a measured signal has parallels with outlier detection [41]. While in applied statistics, the multivariate t-distribution is well established and thoroughly treated in [42]-[44], it has just recently found interest in fault diagnosis and statistical change detection. In contrast to the Gaussian distribution, the t-distribution allows for better modelling of heavy tailed data. Heavy tailed data occur in cases of commonly expected outliers, which is often the case in tracking applications [45] or in any application where sudden changes are to be expected. Due to the heavy tail characteristic of t-distributed data, outliers in the data have less impact on the parameter estimates [46]. This allows for a more robust parameter estimation, which is crucial for reliable change detection [38].

For the purpose of drillstring washout detection, [47] first presented statistical change detection on $\mathrm{t}$-distributed data 


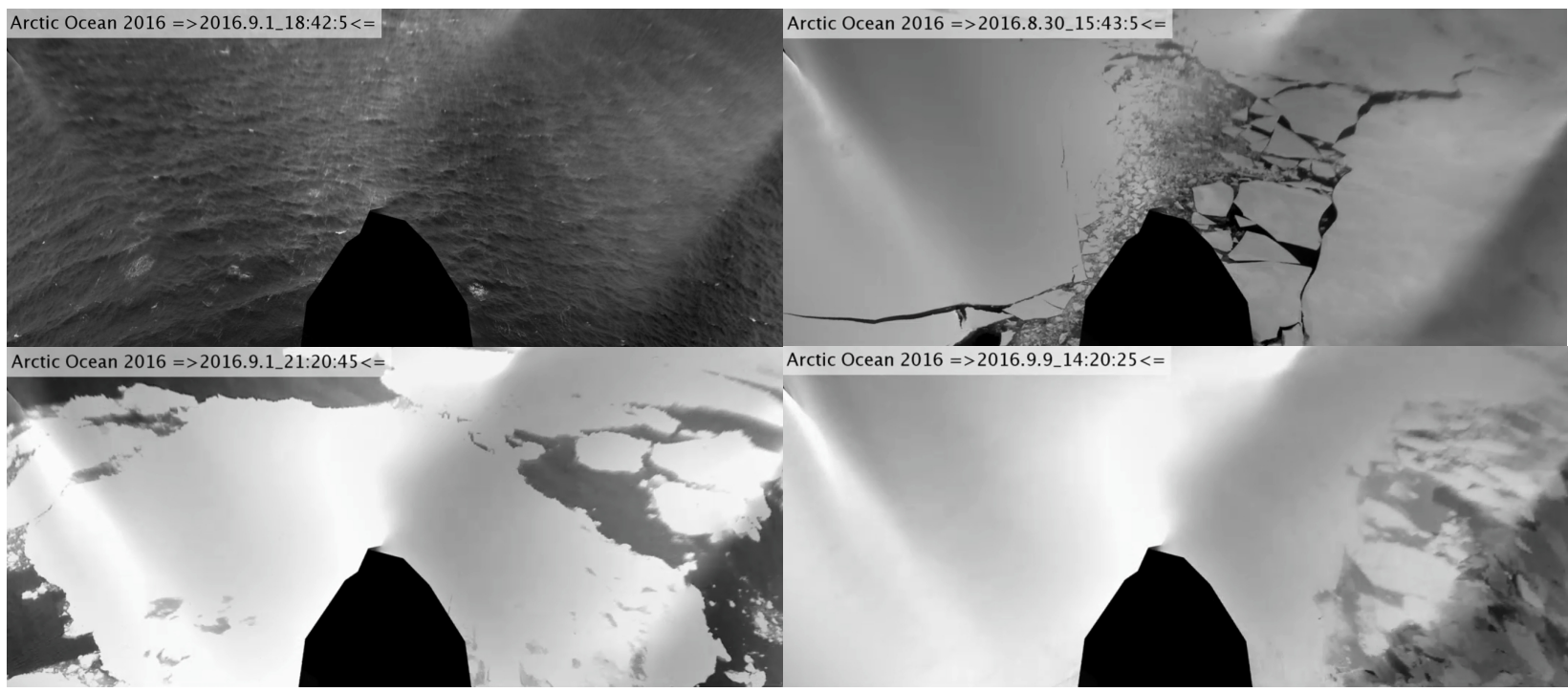

Fig. 3: Pictures of the trained ice conditions, a) Open Water b) Broken ice, c) Close ice d) Very close ice.

using generalised log-likelihood ratio (GLR) testing. The authors derived GLR tests for changes in the mean of univariate and multivariate $\mathrm{t}$-distributed data. They assumed known scale and degrees of freedom parameters, and then they employed maximum likelihood estimators (MLE) for the estimation of the mean in the t-distributed data. For condition monitoring of floating wind turbine drivetrains, [48] showed that statistical change detection for unknown changes in scale and degrees of freedom of a univariate $\mathrm{t}$-distribution can be used. The authors employed both a moment estimator and an MLE for estimation of the unknown parameters of the t-distribution. While a moment estimator for scale and degrees of freedom requires large window sizes for robust estimation and enough degrees of freedom, such that the excess kurtosis is defined $(\nu>4)$, the MLE provided robust estimates, also for short data windows, at the computational cost of solving a set of nonlinear equations.

\section{B. Bivariate t-distribution}

The previous study [28] showed that ice-induced accelerations can be statistically modelled by a univariate t-distribution. This is defined as

$$
f(x ; \mu, s, \nu)=\frac{\Gamma((1+\nu) / 2)}{\Gamma(\nu / 2) s \sqrt{\pi \nu}}\left(1+\frac{(x-\mu)^{2}}{\nu s^{2}}\right)^{-\frac{1+\nu}{2}},
$$

where $\Gamma(x)=\int_{0}^{\infty} t^{z-1} e^{-1} \mathrm{~d} t$ is the gamma function. The three parameters that define the t-distribution are: location $\mu \in \mathbb{R}$, scale $s \in \mathbb{R}$, and degrees of freedom $\nu \in \mathbb{R}$. The univariate t-distribution depends on the results from evaluating the gamma function, which is a computational burden when the parameters for the distribution have to be estimated [49]. To avoid this gamma function evaluation, the following will show how the parameters of a bivariate t-distribution can be estimated with ease if data sets of the $\mathrm{x}$ - and $\mathrm{y}$-acceleration are used together.

The bivariate t-distribution is derived from the joint probability distribution function of a q-variate $\mathrm{t}$-distribution based on the sample data vector $\boldsymbol{x}=\left[\boldsymbol{x}_{1}, \boldsymbol{x}_{2}, \ldots, \boldsymbol{x}_{q}\right]^{\mathrm{T}}$, according to [43]

$$
f(\boldsymbol{x} ; \boldsymbol{\theta})=\frac{\Gamma((q+\nu) / 2)}{\Gamma(\nu / 2)|\boldsymbol{S}|^{\frac{1}{2}}(\pi \nu)^{\frac{q}{2}}}(1+\omega(\boldsymbol{x} ; \boldsymbol{\theta}))^{-\frac{q+\nu}{2}},
$$

with

$$
\omega(\boldsymbol{x} ; \boldsymbol{\theta})=\frac{1}{\nu}(\boldsymbol{x}-\boldsymbol{c})^{\mathrm{T}} \boldsymbol{S}^{-1}(\boldsymbol{x}-\boldsymbol{c}),
$$

where $\boldsymbol{\theta}=[\boldsymbol{c}, \boldsymbol{S}, \nu]^{\mathrm{T}}$ congregates the parameters of the distribution. Furthermore, the mean vector is defined by $c=$ $\left[\mu_{1}, \mu_{2}, \ldots, \mu_{q}\right]^{\mathrm{T}}$, the correlation matrix is given by $\boldsymbol{S} \in \mathbb{R}^{q \times q}$, which contains the scale parameter $\left[s_{1}^{2}, s_{2}^{2}, \ldots, s_{q}^{2}\right]$ of each node on its diagonal entries, and the degrees of freedom of the distribution is $\nu$.

As $\nu \rightarrow \infty$, (9) approaches the multivariate Gaussian distribution [49]. Therefore, the t-distribution is also suitable, as an approximation of the Gaussian distribution for large $\nu$.

The $\mathrm{x}$-acceleration and $\mathrm{y}$-acceleration data can be considered as a sequence of two data sets $\boldsymbol{x}_{1}$ and $\boldsymbol{x}_{2}$, whose correlation is described by a correlation coefficient $\rho$. Each data set contains a sequence of independent random variables with $\boldsymbol{x}_{1} \sim t\left(\mu_{1}, s_{1}, \nu\right), \boldsymbol{x}_{2} \sim t\left(\mu_{2}, s_{2}, \nu\right)$. The joint $\mathrm{t}-$ distribution (9) of $\boldsymbol{z}=\left[\boldsymbol{x}_{1}, \boldsymbol{x}_{2}\right]$ with $q=2$ and

$$
\boldsymbol{c}=\left[\mu_{1}, \mu_{2}\right]^{\mathrm{T}},
$$

is a bivariate $\mathrm{t}$-distribution, given by

$$
f(\boldsymbol{z} ; \boldsymbol{\theta})=\frac{\Gamma(1+\nu / 2)}{\Gamma(\nu / 2)|\boldsymbol{S}|^{\frac{1}{2}}(\pi \nu)}(1+\omega(\boldsymbol{z} ; \boldsymbol{\theta}))^{-\frac{2+\nu}{2}} .
$$




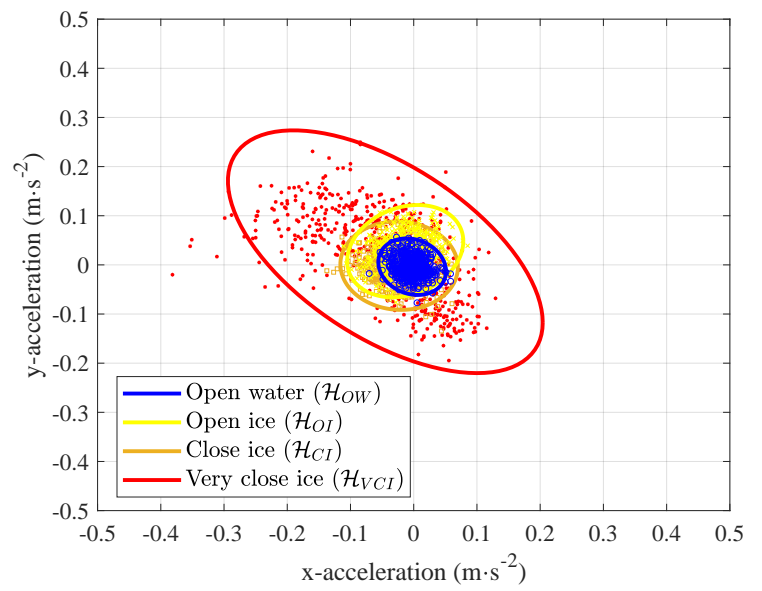

(a)

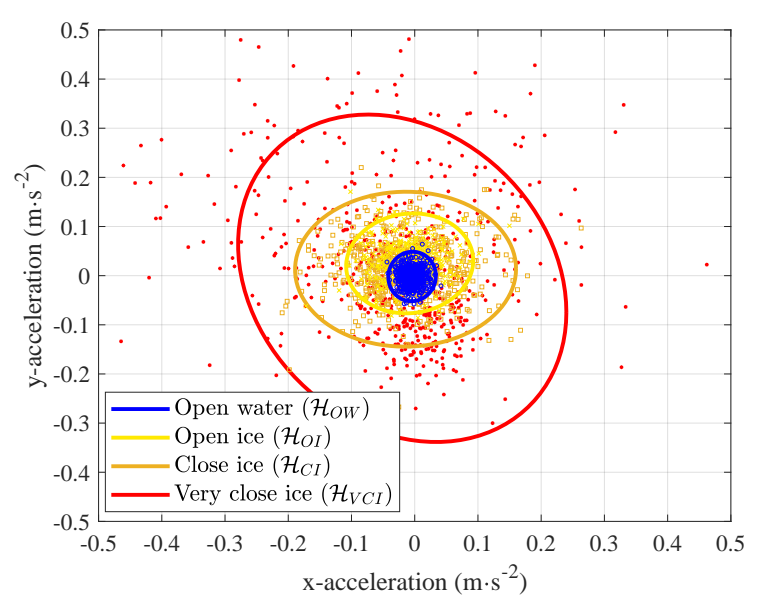

(b)

Fig. 4: Scatter plots of 30 seconds of acceleration data from (a) IMU 1, and (b) IMU 2 under each considered ice condition.

Using the fact that

$$
\frac{\Gamma(1+\nu / 2)}{\Gamma(\nu / 2)(\pi \nu)}=\frac{1}{2 \pi}
$$

turns the evaluation of (12) particularly simple, since the Gamma function evaluation vanishes,

$$
f(\boldsymbol{z} ; \boldsymbol{\theta})=\frac{1}{2 \pi|\boldsymbol{S}|^{\frac{1}{2}}} \cdot(1+\omega(\boldsymbol{z} ; \boldsymbol{\theta}))^{-\frac{2+\nu}{2}},
$$

where the correlation matrix of the $\mathrm{x}$ and $\mathrm{y}$ accelerations is described by

$$
\boldsymbol{S}=\left[\begin{array}{cc}
s_{1}^{2} & \rho s_{1} s_{2} \\
\rho s_{1} s_{2} & s_{2}^{2}
\end{array}\right]
$$

The mean vector $c$, which is caused by accelerometer bias, remains constant and very small over short windows of time. It

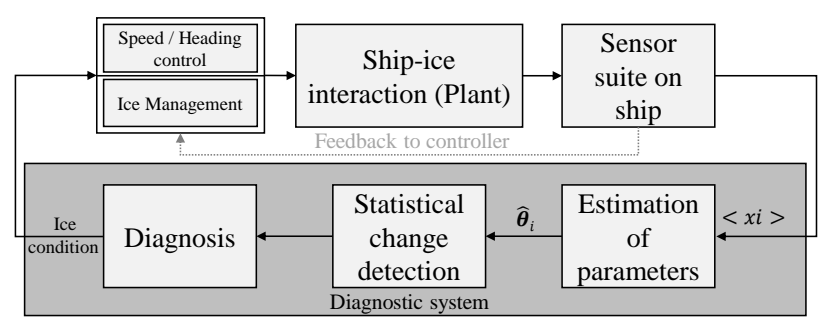

Fig. 5: Structure of an ice diagnostic system.

TABLE IV: Parameter estimates for the bivariate t-distributions under different ice conditions at constant speed (4 knots).

\begin{tabular}{rccccc}
\hline Condition (p) & $\mu$ & $s_{1, p}$ & $s_{2, p}$ & $\rho_{p}$ & $\nu_{p}$ \\
\hline Open water (OW) & 0 & $1.5 \cdot 10^{-4}$ & $2.8 \cdot 10^{-4}$ & $1 \cdot 4.7^{-6}$ & 20.0 \\
Open ice (OI) & 0 & $1.1 \cdot 10^{-3}$ & $1.1 \cdot 10^{-3}$ & $6.3 \cdot 10^{-5}$ & 9.5 \\
Close ice (CI) & 0 & $5 \cdot 10^{-3}$ & $4.5 \cdot 10^{-3}$ & $6 \cdot 10^{-4}$ & 8 \\
Very close ice (VCI) & 0 & $0.7 \cdot 10^{-2}$ & $1.2 \cdot 10^{-2}$ & $2.0 \cdot 10^{-3}$ & 2.8 \\
\hline
\end{tabular}

is therefore only the scale matrix $\boldsymbol{S}$ and the degrees of freedom $\nu$ that change with ice condition. By removing all biases and the influence of the propulsion from the measurements, the mean vector is assumed zero under all ice conditions. The parameters are congregated in $\boldsymbol{\theta}_{p}=\left[\mathbf{0}, \boldsymbol{S}_{p}, \nu_{p}\right]^{\mathrm{T}}$ for each of the trained ice condition out of the set $p \in\{\mathrm{OW}, \mathrm{OI}, \mathrm{CI}, \mathrm{VCI}\}$; see Table IV. Note that the correlation $\rho_{p}$ between the $\mathrm{X}$ and $\mathrm{y}$ accelerations is close to zero in all cases. We assume that the ice-induced vibrations measured by the bow sensor clearly dominates any wind-induced vibrations. Furthermore, we assume that waves can be neglected in the presence of sea ice. Therefore, the parameters in Table IV are assumed to be independent of weather conditions.

\section{Maximum likelihood estimation of parameters}

The parameters $S$ and $\nu$ are estimated from data. Several methods are available among which a maximum likelihood estimation approach was found to be convenient. This estimator is derived as follows: Because an online estimation of the ice conditions is desired, a sliding window of length $N$ selects a sequence $z_{i}^{k}$ with $i=k-N+1$ from the accelerometer measurements. The log-likelihood function for (13) is then

$$
\begin{aligned}
\mathcal{L}_{i}^{k}(\boldsymbol{S}, \nu)= & \sum_{j=1}^{N} \ln f\left(\boldsymbol{z}_{i}^{k}(j) ; \boldsymbol{\theta}\right) \\
= & \sum_{j=1}^{N} \ln |\boldsymbol{S}|^{-\frac{1}{2}}\left(1+\omega\left(\boldsymbol{z}_{i}^{k}(j) ; \boldsymbol{\theta}\right)\right)^{-\frac{2+\nu}{2}} \\
= & -\frac{N}{2} \cdot \ln |\boldsymbol{S}| \\
& -\frac{2+\nu}{2} \cdot \sum_{j=1}^{N} \ln \left(1+\omega\left(\boldsymbol{z}_{i}^{k}(j) ; \boldsymbol{\theta}\right)\right)
\end{aligned}
$$


The set of parameters $\boldsymbol{\theta}_{p}$ can be estimated by solving the maximisation problem

$$
\hat{\boldsymbol{\theta}}=\underset{\boldsymbol{S}, \nu}{\arg \max } \mathcal{L}_{i}^{k}(\boldsymbol{S}, \nu)
$$

To find the maximum of this expression, the derivatives of (16) with respects to $S$ and $\nu$ have to be computed, and the resulting set of two equations has to be equated to zero. With $\boldsymbol{c}=[0,0]^{\mathrm{T}}$, the following set of equations derived from

$$
\frac{\partial \mathcal{L}_{i}^{k}(\boldsymbol{S}, \nu)}{\partial \boldsymbol{S}}=0
$$

gives

$$
\begin{aligned}
& -\frac{N}{2} \cdot \operatorname{tr}\left(\boldsymbol{S}^{-1}\right) \\
& +\frac{2+\nu}{2} \cdot \sum_{j=1}^{N} \frac{\boldsymbol{z}_{i}^{k}(j)^{\mathrm{T}} \boldsymbol{S}^{-2} \boldsymbol{z}_{i}^{k}(j)}{\nu_{p}+\boldsymbol{z}_{i}^{k}(j)^{\mathrm{T}} \boldsymbol{S}^{-1} \boldsymbol{z}_{i}^{k}(j)}=0,
\end{aligned}
$$

and from

$$
\frac{\partial \mathcal{L}_{i}^{k}(\boldsymbol{S}, \nu)}{\partial \nu}=0
$$

gives

$$
\begin{aligned}
& \sum_{j=1}^{N}-\frac{1}{2} \ln \left(1+\frac{1}{\nu} \boldsymbol{z}_{i}^{k}(j)^{\mathrm{T}} \boldsymbol{S}^{-1} \boldsymbol{z}_{i}^{k}(j)\right) \\
& +\frac{2+\nu}{2} \cdot \frac{\boldsymbol{z}_{i}^{k}(j)^{\mathrm{T}} \boldsymbol{S}^{-1} \boldsymbol{z}_{i}^{k}(j)}{\nu\left(\boldsymbol{z}_{i}^{k}(j)^{\mathrm{T}} \boldsymbol{S}^{-1} \boldsymbol{z}_{i}^{k}(j)+\nu\right)}=0 .
\end{aligned}
$$

To obtain the estimate $\hat{\boldsymbol{\theta}}=[\mathbf{0}, \hat{\boldsymbol{S}}, \hat{\nu}]^{\mathrm{T}}$, the set of equations (18) and (19) has to be solved using a nonlinear solver as described in [50]. This result is the maximum likelihood estimate of the parameters that describe the distribution of data within a time window $\boldsymbol{z}_{i}^{k}$ of $N$ samples.

\section{METHODS FOR ICE CONDITION ASSESSMENT}

This section will introduce methods for assessment of ice condition based on statistical measures. A hypothesis test based on the Kullback-Leibler divergence is introduced to identify the condition out of the set of trained ice conditions, which has most similar distribution to the one observed. An alternative approach is the direct comparison of the signal's statistical entropy, which does not require trained data for comparison. Finally, two supervised machine learning approaches using a decision tree model and quadratic support vector machines allow for a benchmark between the two proposed custom methods and the two "off-the-shelf" machine learning approaches.

\section{A. Hypothesis testing}

The ice conditions during a sequence of $N$ observations collected in a vector $\boldsymbol{z}_{i}^{k}$ with $i=k-N+1$ are characterised by a bivariate t-distribution with an estimated parameter set $\hat{\boldsymbol{\theta}}$.
TABLE V: Decision thresholds for hypothesis testing $\left(h_{\mathcal{G}}\right)$ and for entropy based classification $(H(\theta))$.

\begin{tabular}{rcc}
\hline Condition $(\mathrm{p})$ & $h_{\mathcal{G}}$ & $H(\boldsymbol{\theta})$ \\
\hline Open water (OW) & $9.55 \cdot 10^{3}$ & $\geq-12$ \\
Open ice (OI) & $1.12 \cdot 10^{3}$ & $\geq-5.0$ \\
Close ice (CI) & $0.57 \cdot 10^{3}$ & $\geq-3.2$ \\
Very close ice (VCI) & $0.07 \cdot 10^{3}$ & $\geq-1.8$ \\
\hline
\end{tabular}

For each trained set of distribution parameters $\boldsymbol{\theta}=\left\{\boldsymbol{\theta}_{p}\right\}$, the distribution under the observed data is

$$
\begin{aligned}
& \mathcal{H}_{p}: \sum_{j=1}^{k} f\left(\boldsymbol{z}_{i}^{k}(j) ; \boldsymbol{\theta}_{p}\right) \\
& p \in \mathcal{P}=\{\mathrm{OW}, \mathrm{OI}, \mathrm{CI}, \mathrm{VCI}\}
\end{aligned}
$$

and the distribution with the estimated parameter set $\hat{\boldsymbol{\theta}}$ :

$$
\sum_{j=1}^{k} f\left(\boldsymbol{z}_{i}^{k}(j) ; \hat{\boldsymbol{\theta}}\right)
$$

The problem is to find the trained ice condition $p$ out of the set of trained conditions $\mathcal{P}$ that describes best the ice condition characterised by $\hat{\boldsymbol{\theta}}$. Each trained ice condition is described by a bivariate t-distribution with parameter set $\boldsymbol{\theta}_{p}$. A hypothesis test is needed to determine which condition $\boldsymbol{\theta}_{p} \in \mathcal{P}$ best describes the observed condition $\hat{\boldsymbol{\theta}}$.

For this purpose, the Kullback-Leibler divergence, see the overview in [51], provides a measure of the divergence between two probability distributions. Calculating the divergence between the current estimate with the parameter $\hat{\boldsymbol{\theta}}$ and any of the trained distributions with parameters $\boldsymbol{\theta}_{p}$, gives:

$$
D_{k l, p}\left(k, \hat{\boldsymbol{\theta}} \mid \boldsymbol{\theta}_{p}\right)=\sum_{j=1}^{N} f\left(\boldsymbol{z}_{i}^{k}(j) ; \hat{\boldsymbol{\theta}}\right) \ln \frac{f\left(\boldsymbol{z}_{i}^{k}(j) ; \hat{\boldsymbol{\theta}}\right)}{f\left(\boldsymbol{z}_{i}^{k}(j) ; \boldsymbol{\theta}_{p}\right)}
$$

The Kullback-Leibler divergence is 0 when the two distributions are identical.

If a trained ice condition $\boldsymbol{\theta}_{p}$ describes the current ice conditions well, then $D_{k l}\left(k, \hat{\boldsymbol{\theta}} \mid \boldsymbol{\theta}_{p}\right)$ will be a small positive number. To find the trained ice condition that best fits the observation, (22) is used as follows:

$$
\begin{aligned}
D_{k l}\left(k, \hat{\boldsymbol{\theta}} \mid \boldsymbol{\theta}_{p}\right) & =\min _{p \in \mathcal{P}} \sum_{j=1}^{N} f\left(\boldsymbol{z}_{i}^{k}(j) ; \hat{\boldsymbol{\theta}}\right) \ln \frac{f\left(\boldsymbol{z}_{i}^{k}(j) ; \hat{\boldsymbol{\theta}}\right)}{f\left(\boldsymbol{z}_{i}^{k}(j) ; \boldsymbol{\theta}_{p}\right)} \\
& =\min _{p \in \mathcal{P}} G_{i}^{k}\left(\hat{\boldsymbol{\theta}}, \boldsymbol{\theta}_{p}\right) .
\end{aligned}
$$

Selection of Hypothesis: The minimisation over $p$ in (23) finds the training distribution that is closest to the actual sequence of observations. Thus, the most likely condition can be communicated by evaluating:

$$
p(k)=\underset{p \in \mathcal{P}}{\arg \min } D_{k l}\left(k, \hat{\boldsymbol{\theta}} \mid \boldsymbol{\theta}_{p}\right) .
$$


Equation (25) always provides a result that indicates which of the pre-trained conditions are closest, in terms of divergence measure, to the observed distribution. If two of the pre-trained conditions have the same divergence to the distribution obtained during the latest time window, the more severe condition is decided.

Test statistic: The test statistic can be evaluated using (13):

$$
\frac{f\left(\boldsymbol{z}_{i}^{k}(j) ; \hat{\boldsymbol{\theta}}\right)}{f\left(\boldsymbol{z}_{i}^{k}(j) ; \boldsymbol{\theta}_{p}\right)}=\frac{\left|\boldsymbol{S}_{p}\right|^{\frac{1}{2}} \cdot\left(1+\omega\left(\boldsymbol{z}_{i}^{k}(j) ; \hat{\boldsymbol{\theta}}\right)\right)^{-\frac{2+\hat{\nu}}{2}}}{|\hat{\boldsymbol{S}}|^{\frac{1}{2}} \cdot\left(1+\omega\left(\boldsymbol{z}_{i}^{k}(j) ; \boldsymbol{\theta}_{p}\right)\right)^{-\frac{2+\nu p}{2}}},
$$

where $\omega\left(\boldsymbol{z}_{i}^{k}(j) ; \boldsymbol{\theta}\right)$ is defined in (10).

$$
\begin{aligned}
G_{i}^{k}\left(\hat{\boldsymbol{\theta}}, \boldsymbol{\theta}_{p}\right) & =\sum_{j=1}^{N} \frac{\left(1+\omega\left(\boldsymbol{z}_{i}^{k}(j) ; \hat{\boldsymbol{\theta}}\right)\right)^{-\frac{2+\hat{\nu}}{2}}}{2 \pi|\hat{\boldsymbol{S}}|^{\frac{1}{2}}} \\
& \cdot \ln \frac{\left|\boldsymbol{S}_{p}\right|^{\frac{1}{2}} \cdot\left(1+\omega\left(\boldsymbol{z}_{i}^{k}(j) ; \hat{\boldsymbol{\theta}}\right)\right)^{-\frac{2+\hat{\nu}}{2}}}{|\hat{\boldsymbol{S}}|^{\frac{1}{2}} \cdot\left(1+\omega\left(\boldsymbol{z}_{i}^{k}(j) ; \boldsymbol{\theta}_{p}\right)\right)^{-\frac{2+\nu_{p}}{2}}}
\end{aligned}
$$

It can be of interest to know how well an estimated distribution is separated from the other pre-trained conditions. This information can be conducted by applying a supplemental threshold test. With a threshold $h$, the hypothesis test can be supplemented with the following test:

For each $p \in \mathcal{P}$

$$
\begin{array}{llll}
\text { if } & D_{k l}\left(k, \hat{\boldsymbol{\theta}} \mid \boldsymbol{\theta}_{p}\right) \leq h_{\mathcal{G}} & \text { accept } & \mathcal{H}_{p} \\
\text { if } & D_{k l}\left(k, \hat{\boldsymbol{\theta}} \mid \boldsymbol{\theta}_{p}\right)>h_{\mathcal{G}} & \text { accept } & \mathcal{H}_{\neq p} .
\end{array}
$$

Figure 6 shows the results of (27) for all combinations of trained ice condition and the entire set of trained conditions, which are represented by the $\mathrm{t}$-distributions with estimated parameters for each condition. A threshold $h_{\mathcal{G}}$ can be determined for each ice condition to clearly separate the correct ice condition from any other of the trained conditions. The procedure of selecting a value of $h_{\mathcal{G}}$ is described in Appendix B.

This section has shown how an estimate of the distribution, based on horizontal acceleration data over a time window, can be compared with pre-trained data, and a multiple hypothesis test can determine which of the pre-trained conditions are closest to the one observed. A threshold method was suggested as a supplemental means to determine how well the observed data fit one of the trained conditions. When pre-trained data are not available, ice load level assessment can be made anyway, using a measure of statistical entropy. This approach is elaborated next.

\section{B. Condition assessment through statistical entropy}

The Kullback-Leibler divergence is closely related to the statistical entropy [51]. The statistical entropy is a measure on the extent a single probability distribution concentrates on a few points or disperses over many points [42]. Figure $4 \mathrm{~b}$ shows how the dispersion of datapoints depends on the ice condition.
The differential entropy for a random variable $\mathbf{Z}$ with probability function $f\left(\boldsymbol{z}_{i}^{k}(j) ; \boldsymbol{\theta}\right)$, data sequence $\boldsymbol{z}_{i}^{k}(j)$ of length $N$ with $i=k-N+1$, and parameter vector $\boldsymbol{\theta}$, is defined by [52] as

$$
H(\mathbf{Z})=-\sum_{j=1}^{N} f\left(\boldsymbol{z}_{i}^{k}(j) ; \boldsymbol{\theta}\right) \ln f\left(\boldsymbol{z}_{i}^{k}(j) ; \boldsymbol{\theta}\right)
$$

For a q-variate, central $(c=0)$ t-distribution (9), and the parameter estimate $\hat{\boldsymbol{\theta}}=[\mathbf{0}, \hat{\boldsymbol{S}}, \hat{\nu}]^{\mathrm{T}}$, the entropy solely depends on the parameter estimate $\hat{\boldsymbol{\theta}}$ and is given by [53] as

$$
\begin{aligned}
H(\hat{\boldsymbol{\theta}}) & =\frac{1}{2} \ln |\hat{\boldsymbol{S}}| \\
& +\ln \left(\frac{(\hat{\nu} \pi)^{\frac{q}{2}}}{\Gamma\left(\frac{q}{2}\right)} B\left(\frac{q}{2}, \frac{\hat{\nu}}{2}\right)\right) \\
& +\frac{q+\hat{\nu}}{2}\left(\psi\left(\frac{q+\hat{\nu}}{2}\right)-\psi\left(\frac{\hat{\nu}}{2}\right)\right),
\end{aligned}
$$

where $\psi(x)=\mathrm{d} \ln (\Gamma(x)) / \mathrm{d} x$ denotes the digamma function, and $B(x, y)=\Gamma(x) \Gamma(y) \Gamma(x+y)^{-1}$ denotes the beta function.

For a bivariate t-distribution $(q=2)$, the expression (30) can be significantly simplified, using $\Gamma(x+1)=x \Gamma(x)$, and $\psi(x+1)=\psi(x)+x^{-1}$ to give:

$$
H(\hat{\boldsymbol{\theta}})=\frac{1}{2} \ln |\hat{\boldsymbol{S}}|+\ln (2 \pi)+\left(\frac{\hat{\nu}}{\hat{\nu}+2}\right) .
$$

Figure 7 shows the entropy for the four different ice conditions of Figure 3. The ice conditions can be discriminated by defining entropy thresholds using the training data set. Those entropy specific thresholds are given in Table V. The statistical entropy is seen to associate a monotonic increasing grade to ice condition. Being an absolute measure related to measured accelerations, the statistical entropy measure can be extrapolated beyond the range of pre-classified conditions, and it can express a condition in between those that were pre-classified. The expression (31) is computationally efficient.

\section{Algorithm for ice condition assessment}

The following algorithm summarises the proposed methods for ice condition assessment: 


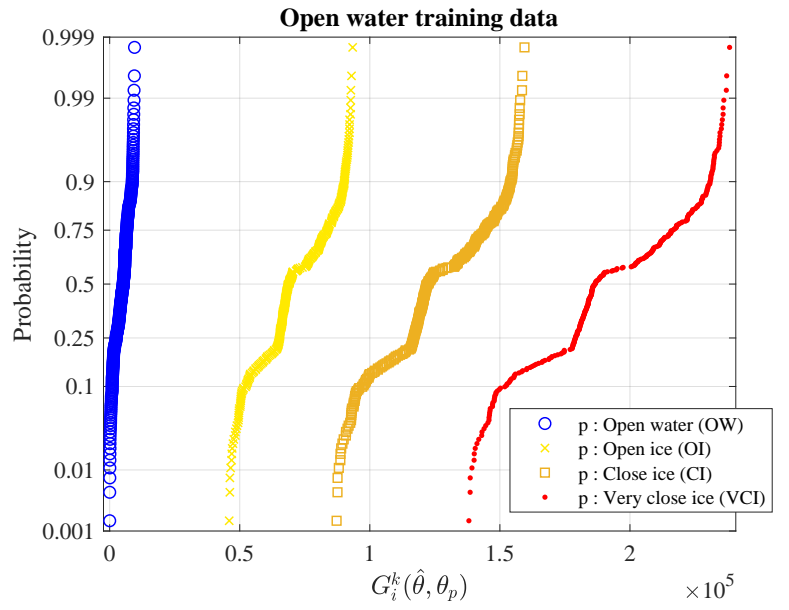

(a) Test statistic for trained open water parameter set

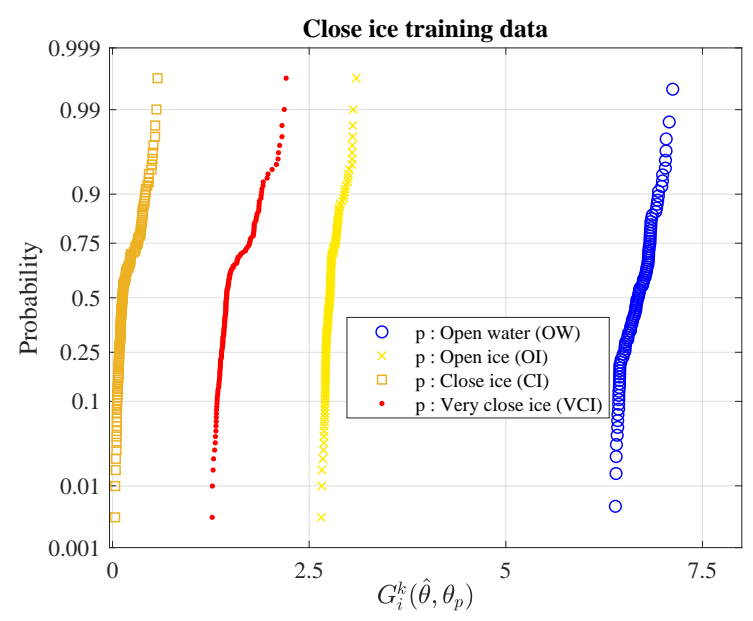

(c) Test statistic for trained close ice parameter set

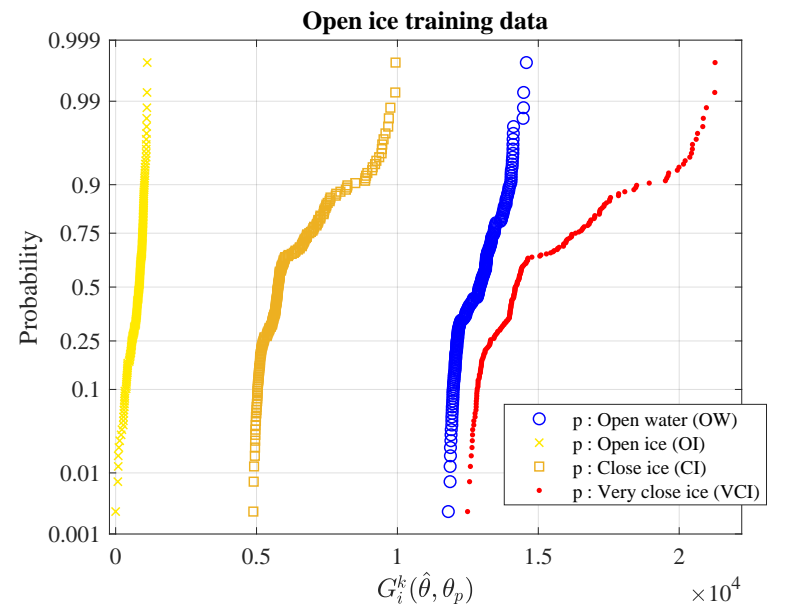

(b) Test statistic for trained open ice parameter set

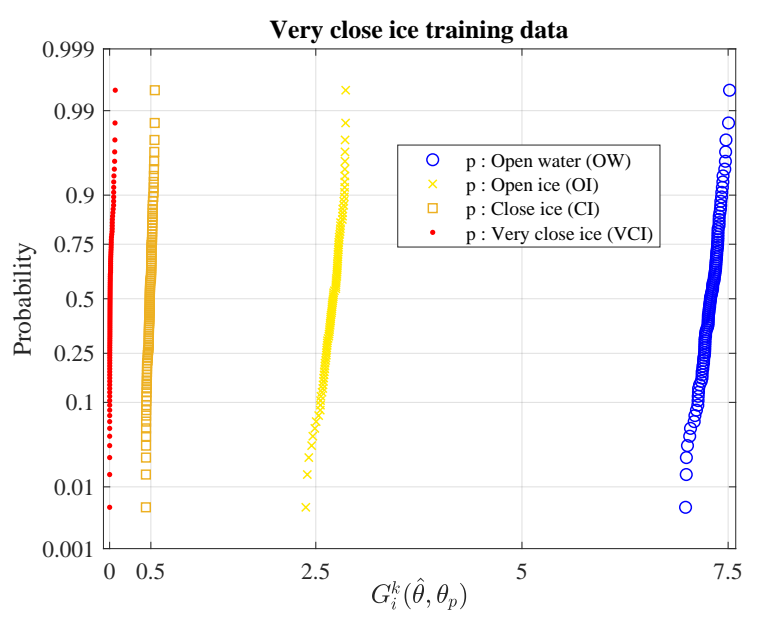

(d) Test statistic for trained very close ice parameter set

Fig. 6: Probability plots of the result from the test statistic (27) with different data sets under each trained ice condition.

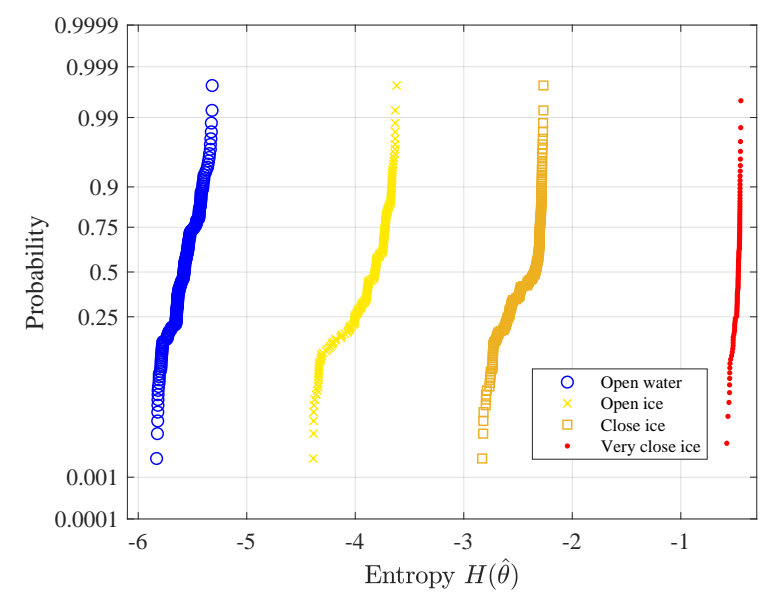

Fig. 7: Probability plot of the entropy results under the four training data ice conditions. 


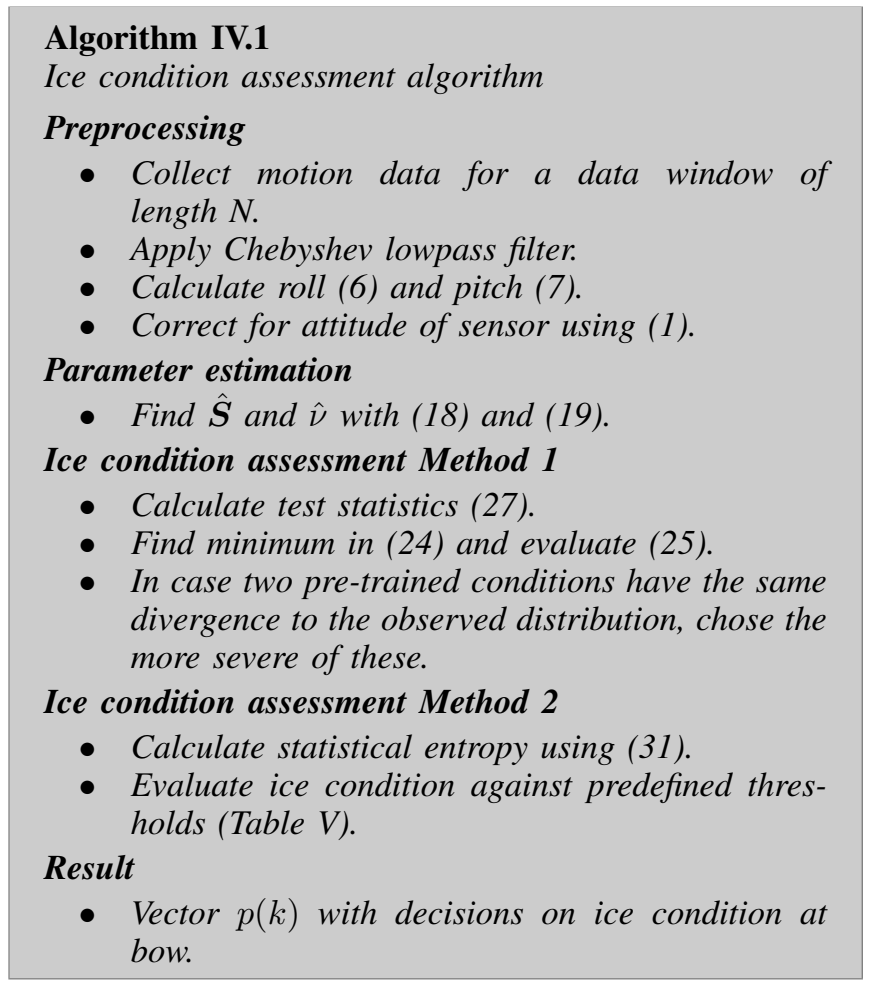

\section{Machine learning approaches}

Two standard machine learning approaches serve as a benchmark for the statistical classification methods introduced earlier. Both machine learning approaches were trained with the training data presented in Figures 3 and $4 \mathrm{~b}$.

The Matlab ${ }^{\circledR}$ Classification Learner toolbox provides a number of supervised machine learning approach for training of classification models, among others decision tree models, support vector machines, and nearest neighbour classifiers. An overview of classification learning methods is given in [54].

Training data: Using the pure acceleration data as depicted in Figure $4 \mathrm{~b}$ did not result in reasonable classification models. Therefore, the machine learning models were trained with statistical data, specifically the components of the correlation matrix (14) and the degrees of freedom $\nu$ as described in (13). For each considered ice condition, 30 seconds of training data were used, the same length as for the earlier described change detection methods.

Validation: Cross-validation with five folds was used for validation of the trained models. For this approach the training data were partitioned into five disjoint sets. For each set the model was trained with the data which were not part of the specific set. The data in the remaining disjoint sets were used for validation. The process was repeated for each set. The best training results were archived with a decision tree model and a quadratic state-vector machine.

Decision Tree model: Decision Trees are a popular methods for classification models [55]. A detailed survey on the construction of decision trees can be found in [56]. Figure 8 illustrates the trained decision tree for ice condition classification.

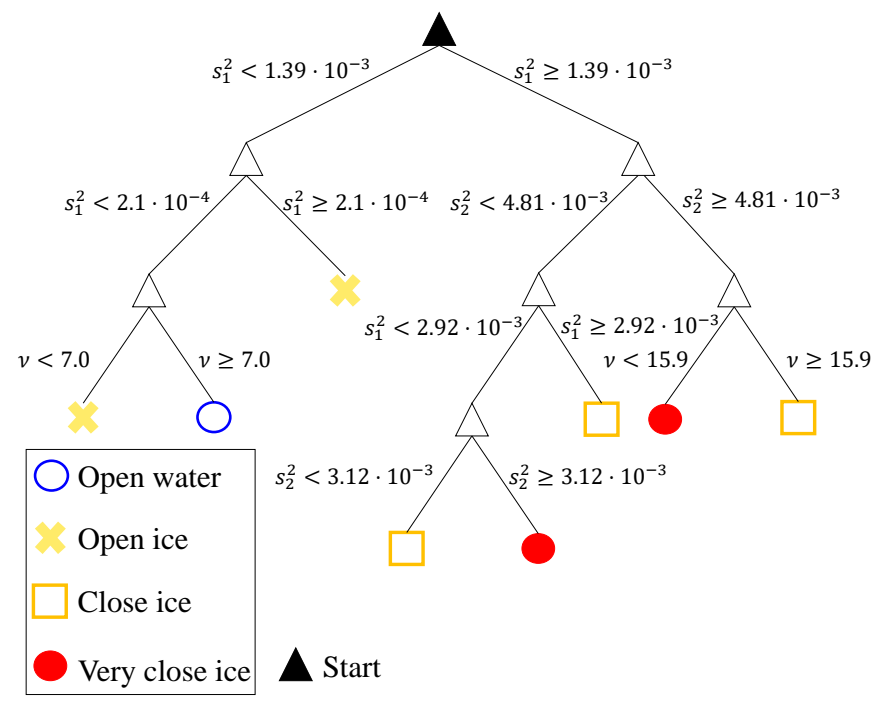

Fig. 8: Trained decision tree for ice condition detection

Support vector machine: Support vector machines try to classify training data by introducing separating hyperplanes which depend on data points lying on margins called support vectors. Maximisation of the margins results in better generalised performance under unknown data; see [57]. A kernel operation can transform the training or input data to a higher dimension feature space, which allows a clearer definition of the margin regions. The best SVMs were obtained using a quadratic kernel and the statistical properties of the training data as input, specifically the results from equations (18) and (19). Figure 9 contains three plots that illustrate the relation between the estimated parameters of the statistical model (12) for each ice condition in the training sets. It is obvious that already with a linear SVM, planes can be constructed to separate the ice conditions well. The quadratic SVM uses a quadratic kernel to create a transformed feature space, allowing for an even better separation of the ice condition regions.

\section{VALIDATION OF CUSTOM CLASSIFICATION METHODS USING FULL SCALE DATA}

For three data sets, recorded during transit in ice, the ice conditions at the bow of the vessel were determined using two custom classification algorithms IV.1 with the trained parameter sets (Table IV) and thresholds (Table V) found with the training data set. The data sets serve as validation for the proposed ice condition assessment system. In all cases, the apparent windspeed and the heading of the vessel were constant during the measurements. The sampling rate of the sensors was decimated to $20 \mathrm{~Hz}$. A sliding window collects measurement samples for processing. The window size determines the detection delay (more delay with larger data window), but also the steadiness of the detection result (more steady results with larger data window). Figure 10 presents the detection results for three different window sizes. As a 


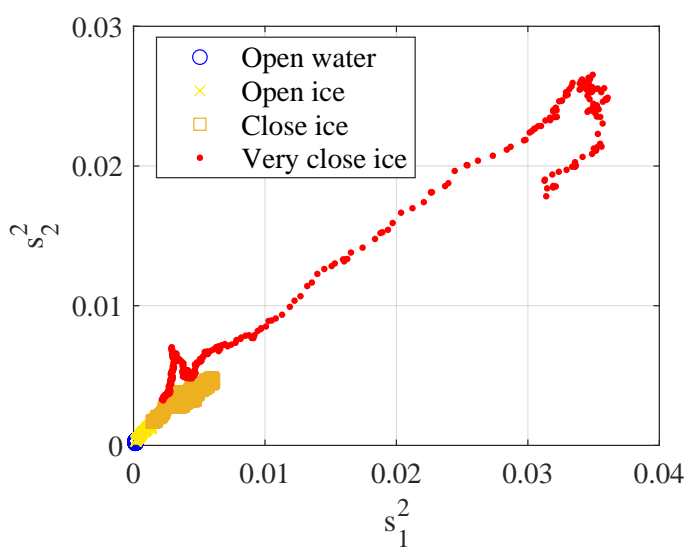

(a)

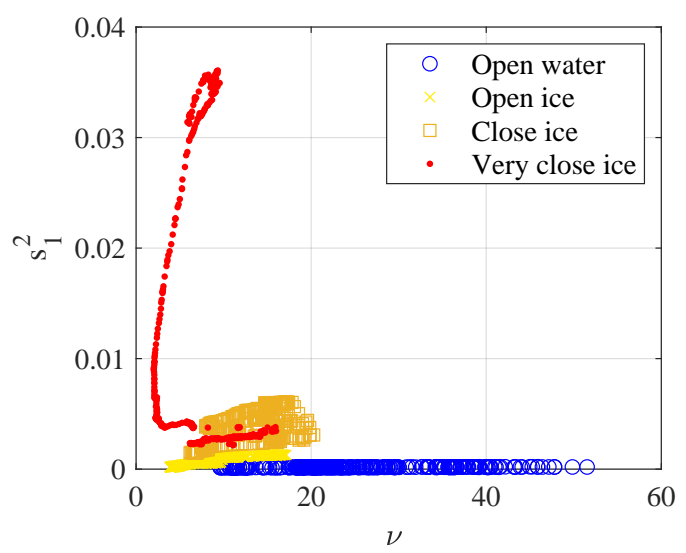

(b)

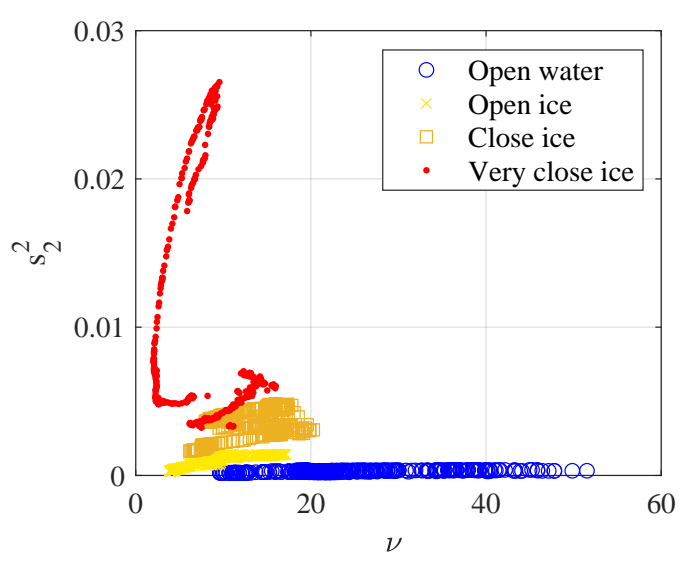

(c)

Fig. 9: Scatterplots of statistical parameters of the training data for model (13) (a) $s_{1}^{2}$ and $s_{2}^{2}$ (b) $\nu$ and $s_{1}^{2}$ (c) $\nu$ and $s_{2}^{2}$. compromise between speed of detection and steadiness of result, a window size of 10 seconds was chosen for this study.

Figures 12 - 14 consist each of four sections:

1) Section (1) shows images from a camera system. The images were taking in synchronisation with the acceleration measurements.

2) Section (2) gives a plot of the ice concentration at the bow of the ship. The ice concentration has been continuously determined (every 5 seconds) from the images using image processing techniques described in [8]. Because the camera system can only differentiate between ice cover and open water, and does not include a measure of other ice properties such as thickness or porosity, the camera analysis is merely an indicator of the ice condition.

3) Section (3) presents the results from the differential entropy based classification of ice conditions. The section contains a plot of the entropy (31), and a colourbar, which indicates the results of the classification using the thresholds defined in Table V.

4) Section (4) gives the results of the hypothesis test. The decision function $G_{i}^{k}\left(\boldsymbol{\theta}_{p}, \hat{\boldsymbol{\theta}}\right)$ for each trained ice condition are plotted, and the result of (25) are illustrated as a colourbar.

\section{A. Case 1: Transit in open water until impact with a single ice floe}

In the scenario shown in Figure 12, the ship travelled at 6 knots in open water. Upon impact with an ice floe at 21:20.30 the speed over ground reduced to 4.5 knots.

Both diagnostic approaches recognise correctly the open water condition at the beginning. Before the impact with the solid ice floe smaller ice pieces were encountered, which both systems classify as open ice condition. Upon impact with the unbroken ice floe, the ship slows down due to the pressure

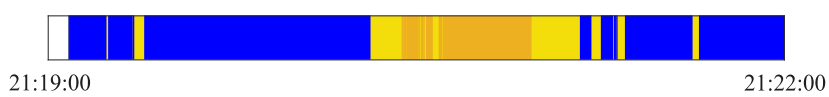

(a) Data window of 5 seconds

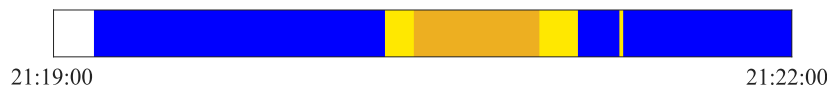

(b) Data window of 10 seconds

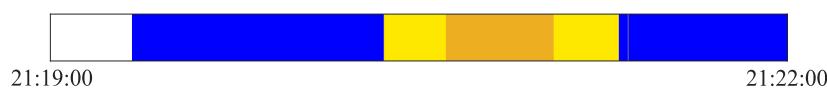

(c) Data window of 20 seconds

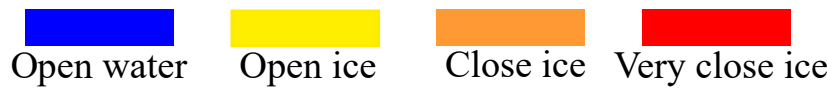

(d) Legend

Fig. 10: Detection results of Case 1 data under hypothesis testing with different data window sizes. 
increase against the hull. Both approaches detect close ice conditions. The pressure against the hull was released when the ice floe broke. This causes the classification of the ice condition to change to open ice. Towards the end of the measurement the ship pushed the broken ice floes away instead of further breaking them. This means that the bow of the vessel travelled in open water, which is correctly assessed by both diagnostic approaches. The entropy based method reacts more sensitive to changes in the ice conditions.

\section{B. Case 2: Transit in broken ice}

Figure 13 shows the diagnostic results during transit at 7.5 knots in broken ice of $30-40 \mathrm{~cm}$ thickness.

At first the ship travelled for a short period in open water, which both methods detect correctly. At 15:39.40 the ship entered broken ice, and both approaches for the diagnostic system correctly classify this as open ice. At 15:39:45 both methods declare the encounter with a smaller unbroken ice floe as close ice. At 15:40:30 the ship encountered several larger ice pieces, which were rammed and split. Both methods declare this as close ice. At 15:41.50 the ship encountered open water followed by a channel of broken ice as shown in the last image of Figure 13. Both system declare the open water correctly. The entropy based method reacts faster on the ice condition change. Compared to the result from the camera system, the entropy based method seems to be more correct, especially because it registers earlier the encounter of unbroken ice pieces at 15:40:30.

\section{Case 3: Transit in close ice}

Figure 14 shows the third scenario in which the ship encountered heavy ice conditions. The ice in this case was thicker $(>50 \mathrm{~cm})$ than in the previous two cases. It consisted mostly of closed level ice with areas of newly frozen ice in between. The ship travelled at 6.5 - 7.5 knots.

Both the entropy and the hypothesis testing methods register close ice conditions at the bow of the vessel in the beginning of the measurement with a short moment of open ice. At 11:29.40, the ship encountered a large, unbroken ice floe (with a diameter of 80-100 metres). It required several seconds until the ice floe broke. During this time the pressure against the hull increased so much that both methods classify the situation as very close ice conditions. The speed of the vessel reduced nearly to zero. At 11:30.25 the ice floe split and the pressure was released. At this point the results from the two approaches deviate slightly. The entropy based approach detects open ice conditions while the hypothesis testing approach detects first close ice conditions. The camera image at 11:31.00 shows an area of newly formed ice, which should not create major resistance against the hull. Therefore the entropy-based result seems correct. Upon inspection of the full camera image several ice pieces starboard of the vessel are visible. These have formed a string and broke simultaneously just before 11:31.00; see Figure 11. This may have caused pressure against the hull. On the image at 11:32.00 an unbroken larger ice floe can be seen ahead of the vessel's bow. At 11:32.35 the ship split the ice floe. Both systems declare this event as very close ice conditions. On the last image a major unbroken ice floe is visible. Forty seconds later the ship hits this ice floe. That causes the ice diagnostic system to declare the situation correctly as very close ice conditions again.
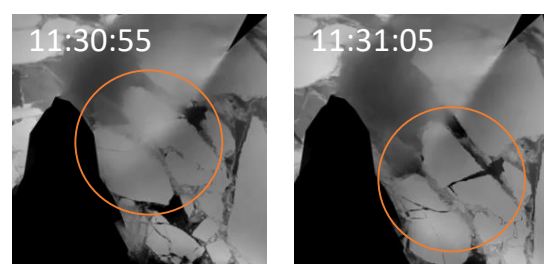

Fig. 11: Simultaneous breaking of ice pieces starboard of vessel at 11:31.00.

\section{Comparison with machine learning approaches}

Figure 15 gives a comparison of the detector results of custom classification methods with the results from the two trained "off-the-shelf" machine learning classification models introduced earlier.

Using the data of Case 1, in which the vessel encountered a larger unbroken ice floe, all methods are capable of detecting the event. During transit in open water the decision tree based classification model often classifies open ice conditions. The SVM model provides more steady results. As an example, Figure 16a provides a ground truth of the ice situation at 21:19:15 (marked with a black line in Figure 15a). The bow was completely surrounded by open water. Therefore, the assessment of the decision tree method is wrong.

Using the data of Case 2, in which the vessel transited in broken ice and encountered an area of open water, the decision tree model works more stable, whereas the SVM model provides more erratic classification during the open ice situation. All methods, however, detect correctly the open water periods. The machine learning based models seem to react faster on changes. Figure $16 \mathrm{~b}$ shows that at 15:41:40 (marked with a black line in Figure 15b) the bow of the vessel was already inside open water. The statistical change detection methods react a few seconds delayed due to the sliding data window. On the other hand, the sliding data window allows for a more steady result compared to the machine learning based models.

Using the data of Case 3, in which the vessel was exposed to more severe ice conditions, all four methods detect all three encounters of large and unbroken ice features (very close ice conditions). The open water patch in the middle of the measurement was thereafter reliably detected by all methods. The SVM based classification gives more erratic results. In the last quarter of the measurement, the decision tree model wrongly classifies open water, where the camera images do show the presence of light ice conditions; see Figure 16c. Although it looks like open water, the bow actually travelled inside thin, unbroken ice just in front of a major unbroken ice floe. Open water appears darker on the image, as it is visible in 


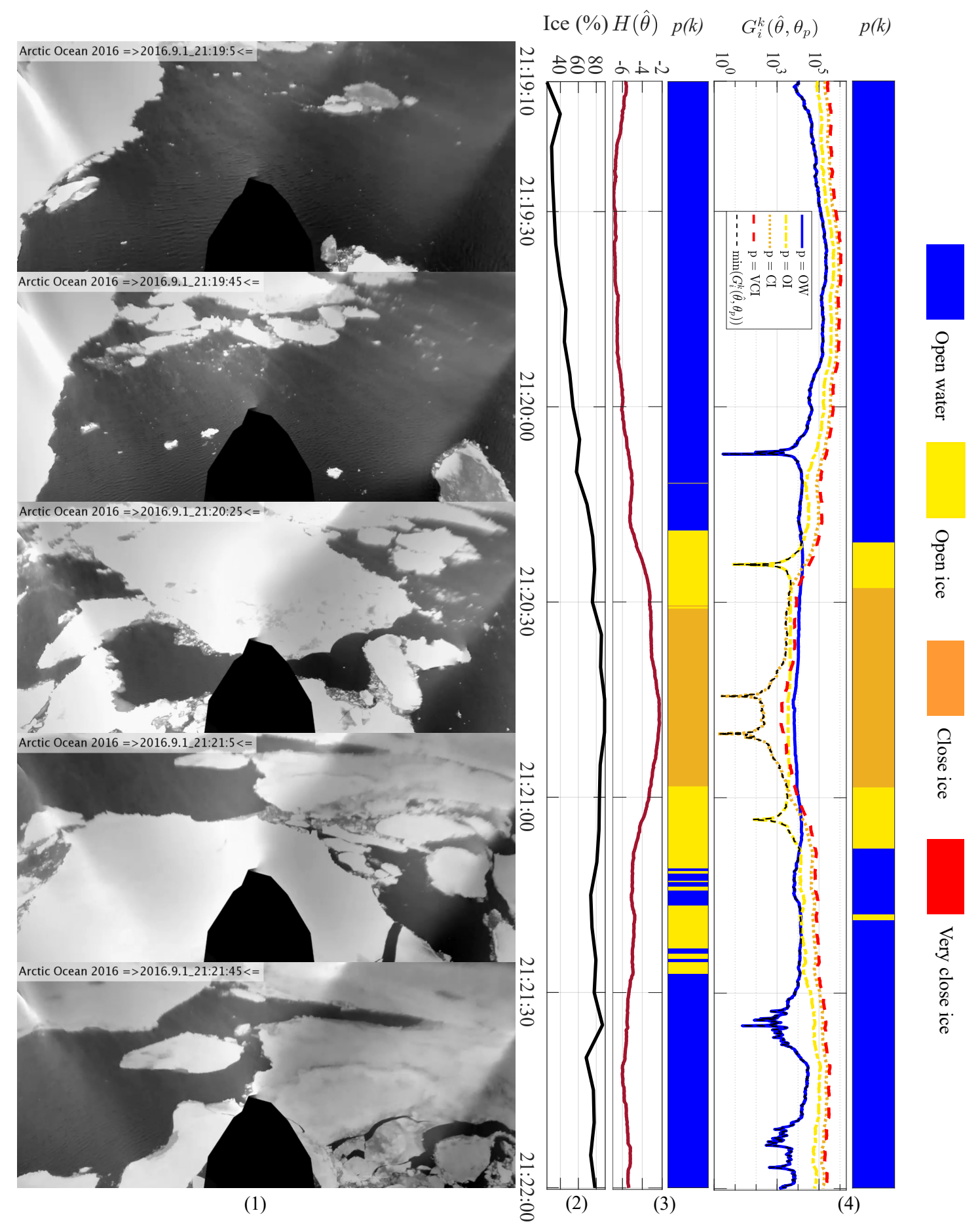

Fig. 12: Test statistic and classification result during transit onto an solid ice floe in open water (Case 1). 


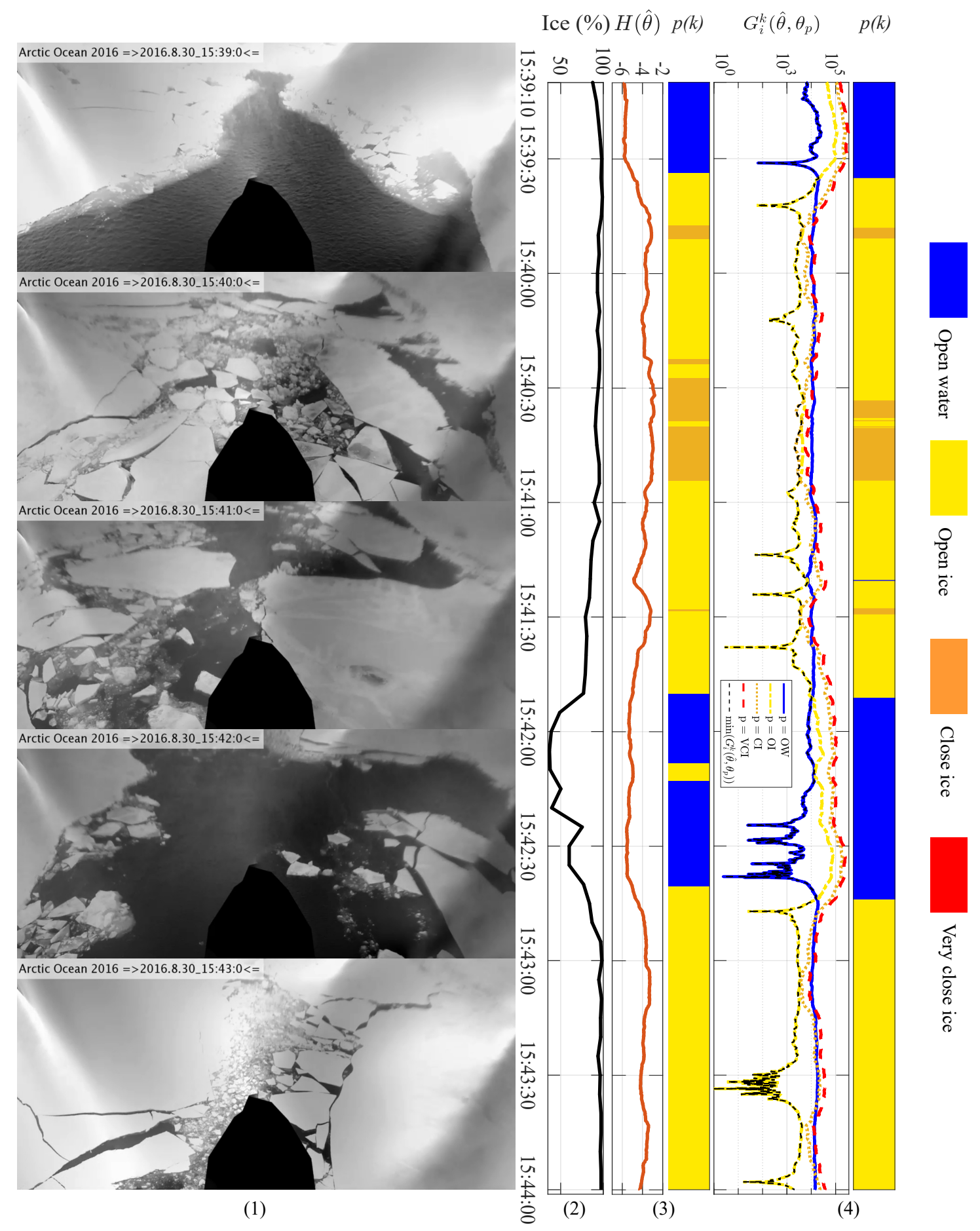

Fig. 13: Test statistic and classification result during transit in broken ice (Case 2). 


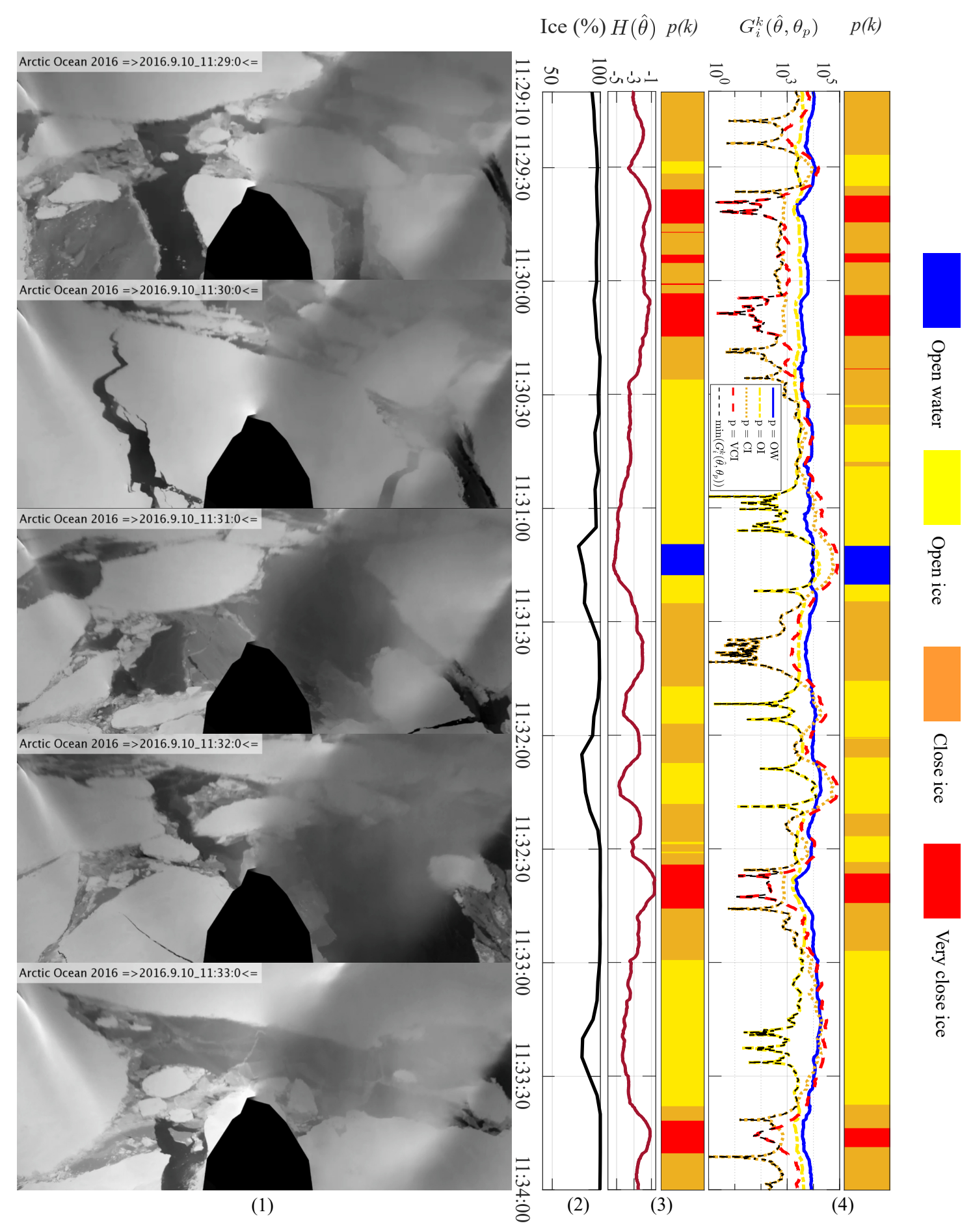

Fig. 14: Test statistic and classification result during transit in close ice (Case 3). 


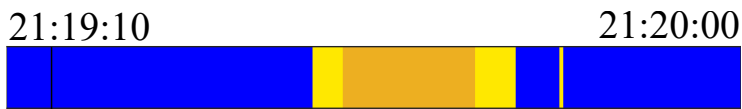

(a) Hypothesis testing

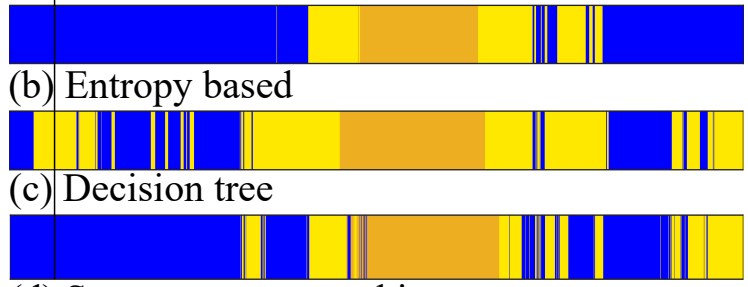

(d) Support vector machine

(a) Comparison of classification results for transit onto solid ice floe in open water (Case 1).

$15: 39: 10$ $15: 44: 00$

(a) Hypothesis testing

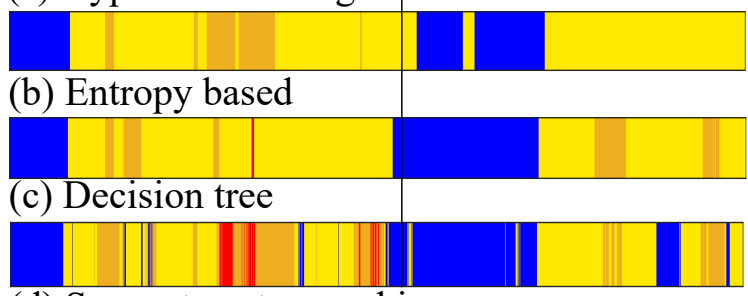

(d) Support vector machine

(b) Comparison of classification results during transit in broken ice (Case 2).

$11: 29: 10$ $11: 34: 00$

(a) Hypothesis testing

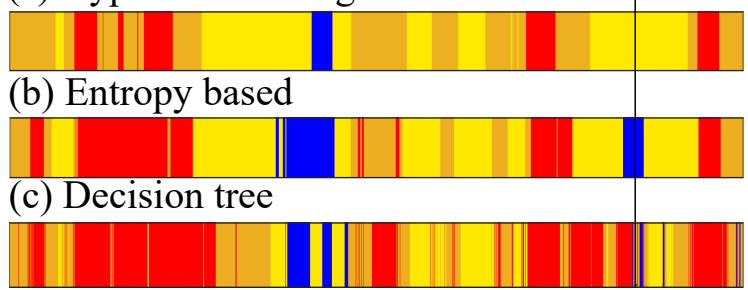

(d) Support vector machine

(c) Comparison of classification results during transit in close ice (Case 3).

\section{Open water Open ice Close ice Very close ice}

(d) Legend

Fig. 15: Comparison of custom classification methods with "off-the-shelf" machine learning approaches.

the left part of the image, where patches of sea water are visible where the thin ice had broken up. Therefore, the assessment of open ice is correct.

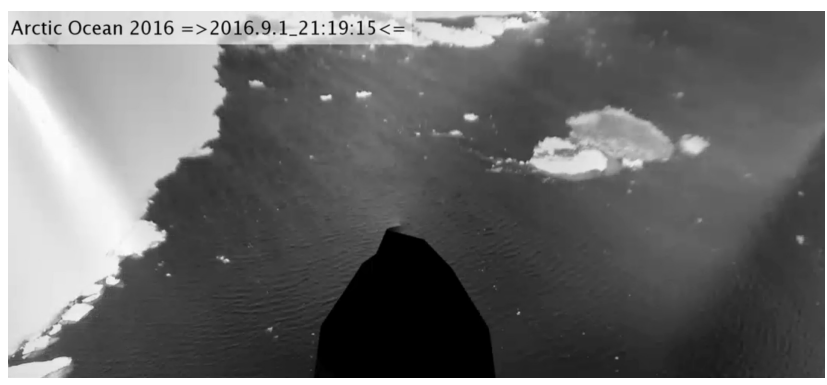

(a) Ice situation Case 1 at 21:19:15

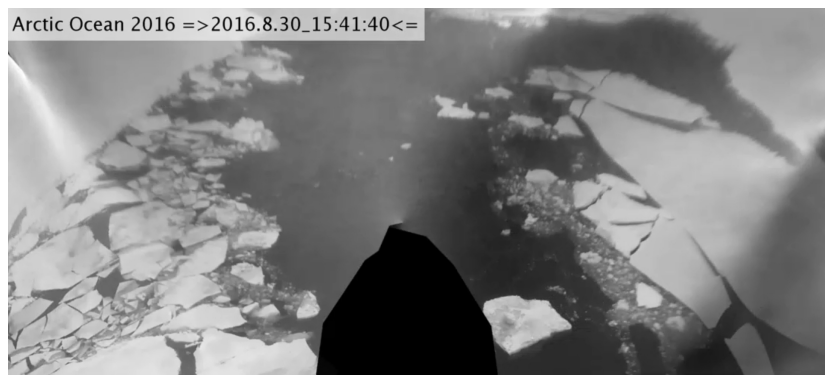

(b) Ice situation Case 2 at 15:41:40

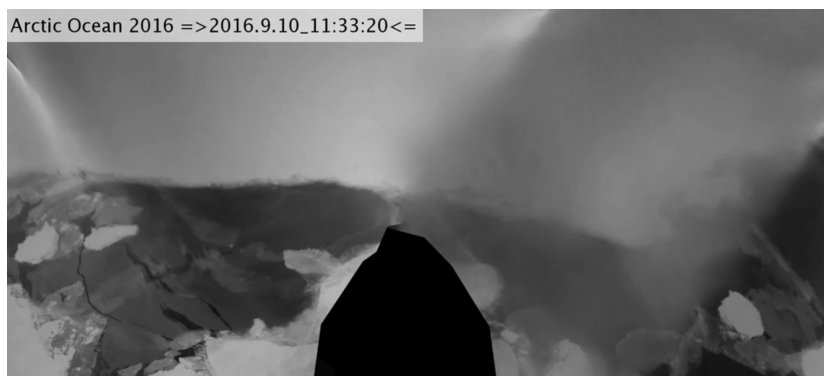

(c) Ice situation Case 3 at 11:30:20

Fig. 16: Use of image proof to determine correct ice condition

\section{Vi. Discussion, Perspectives, And Conclusion}

\section{A. Discussion}

Two custom classification algorithms, that are based on estimating the parameters of the t-distribution of data, were compared with two machine learning methods. The methods have the following characteristics:

- Hypothesis test is made by calculating (27). Training data are needed to represent the categories in which we wish to classify the ice condition. A hypothesis is selected that is closest to fits a category using (25). This study used four categories of ice conditions. Data represented each by parameters of the associated tdistribution. Additional training data can easily be added, should this be preferred.

- Entropy test provides an absolute measure through calculation of (31). The entropy detector can extrapolate to conditions where training data are not available, and can 
interpolate, and can supplement the hypothesis test.

- Two machine learning methods were employed: a decision tree model and a support vector machine model. The training data sets were used for learning. The training of machine learning models to data is transparent and does not physical behaviours are not directly represented in model parametrization.

The comparison of the two statical methods with the two selected approaches from machine learning showed that the statistical methods, being based on inherent knowledge of the underlying statistics of the physical processes, provide steadier results. The statistical tests have the window size is a tuning parameter and more steady, albeit slower, assessment can be achieved by using a larger data window size for parameter estimation.

All methods were able to detect sudden and significant changes of ice conditions, for example the transition from open ice to harsh unbroken ice.

The training of machine learning models require test cases to train correct classification, and it is difficult to control how the models adapt to each ice condition. For example, the automatically trained decision tree model in Figure 8 is unnecessarily complicated and does not follow a physical reasoning.

The statistical classification is based on a physical reasoning: Heavier ice conditions causes more fluctuations of the hull vibrations, and as a result the scale parameter of the $\mathrm{t}$ distribution increases. Furthermore, high energy spikes, caused by the interaction of heavy ice feature, result in a heavytail characteristic of the recorded signal, which results in a low degree of freedom of the estimated t-distribution. An automatically trained machine learning model does not take these physical phenomena into account.

\section{B. Perspective}

The installation of IMUs in the hull of an ice-going ship is very simple. In combination with the proposed diagnostic system, there is a range of applications that this system can be used for:

- The most obvious application is a decision support system for ice-going vessel, especially container vessels travelling the Arctic sea route or cruise ships entering ice-infested waters. The system can support a warning system for getting locked-in ice. The system can also assist icebreakers which scout ice conditions for ice management.

- By distributing a sensor array in the hull of the vessel, a local awareness of the ice conditions around a vessel can be obtained. Although the system has not yet been deployed during stationkeeping in ice, it can be tested if the proposed diagnostic system can detect the accumulation of ice at one side of the stationkeeping vessel; see [58]. This information can be forwarded to a control system, which e.g. changes the heading of the stationkeeping vessel to counteract the accumulation of ice along the hull.
- The system is currently a reactive system. It communicates the ice conditions, which are directly affecting the vessel. However, a deeper statistical analysis using extreme value prediction of occurring ice loads can allow for a forecasting of ice loads.

- This diagnostic system might be extended to differentiate between different sea states in open water, see [59] and references herein. In addition to in-plane accelerometers, data from z-acceleration, angular rates, and roll-pitch motion could be utilised, and a multiple hypothesis test could be designed to decide between different sea states.

\section{Conclusion}

A system for ice condition assessment was developed which solely used data from accelerometers in the hull of an ice-going vessel. Merging the data from the in-plane accelerometers, it was possible to describe the ice-induced accelerations with a bivariate t-distribution. The bivariate $\mathrm{t}$-distribution made it possible to obtain a maximum likelihood estimator without making use of the Gamma function, and it appeared to be very robust. Training data from different ice conditions gave reference parameters of bivariate t-distributions that represented each of the training conditions.

Two principal custom methods were proposed for detection of the prevailing ice condition. A modified Kullback-Leibler divergence formulation was used with a hypothesis testing scheme to classify the current ice condition being closest to a training condition. A second method did not require training data but utilised the change in statistical entropy of the accelerations. It was found that the entropy changes significantly, and in a monotone way with ice conditions, which demonstrated that the entropy measure is a good indicator for ice load. Additionally, a decision tree model and a support vector machine classifier were trained with the same training data as the custom methods.

All classification methods were tested on three data sets, obtained during transit in the Arctic Ocean under different ice conditions. Both statistical classification methods, i.e., hypothesis testing and entropy estimation, produced valid and very similar results for the ice condition assessment, which appeared to agree with the ice conditions judged from simultaneous camera system recordings. Classification using standard machine learning models also produced valid results, but they showed to be more fluctuating.

Working 24/7, being independent of visibility conditions, and providing objective estimates of ice condition, it is concluded that an IMU-based ice condition monitoring system can indeed be a good addition for decision support on vessels operating in ice-infested waters. Installation of the required sensors is easy, the method works robustly, and extrapolation outside training data will be feasible. Further possible applications of the monitoring system were proposed, such as the detection of ice accumulation along the hull of a ship.

\section{APPENDIX A}

\section{STATISTICAL INDEPENDENCE OF MEASUREMENTS}

One assumption is that the measurements are independent. To show that this assumption is justified, Figure 17 

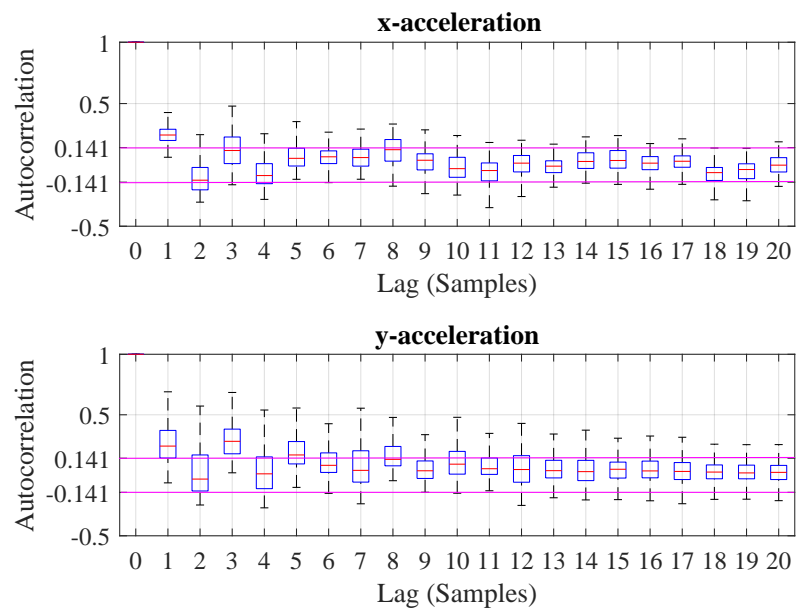

Fig. 17: Correlogram of Case 1 measurements from IMU 2

presents a correlogram of the measurements from Case 1 . The entire dataset contained 3600 datapoints. Because the proposed system operates on data windows of 200 samples, the autocorrelation function was determined for a sliding window of 200 samples at a time. The results are presented as boxplots, showing in red the median value, and the box representing the 25 th to 75 th percentiles. The $95 \%$ confidence interval for assuming statistically independence is indicated as purple line. The correlation plot shows for both $\mathrm{x}$ - and $\mathrm{y}$ accelerations an autocorrelation on the first lag (and third lag for $y$-acceleration) that exceeds independence level. All other lags show an autocorrelation that supports the hypothesis of independence within a 95\% confidence level. Because data originate from a physical process, the assumption of statistical independence may not hold perfectly. One consequence is that the test statistics (27) will not follow a chi-square distribution [40], [47]. The evidence for independence is concluded to be strong enough for our development.

\section{APPENDIX B \\ THRESHOLD SELECTION}

This appendix details how a threshold is determined from test data.

\section{Algorithm B.1 \\ Threshold calculation algorithm.}

Distribution of test statistics

- Given the training datasets $z(k ; p), p \in P$ and the parameter vector $\boldsymbol{\theta}_{p}$ for each training data set.

- Calculate the cumulative distributions $\mathcal{G}_{q, p}$ of the test statistics $\boldsymbol{G}_{i}^{k}\left(\boldsymbol{\theta}_{q}, \boldsymbol{\theta}_{p}\right), q \in P$ and $q \neq p$.

- Approximate each of the cumulative distributions $\mathcal{G}_{q, p}(G)$ by smooth functions $\mathcal{F}_{q, p}(G)$.

- Define a probability of wrong classification $P_{\text {clas }}$ e.g. $10^{-4}$.

- $\forall q, p, q \neq p$, find a threshold $h_{\mathcal{G}}=\max _{h}\left|\mathcal{G}_{q, p}(h)\right| \leq P_{\text {clas }}$.

\section{Result}

- A threshold $h_{\mathcal{G}}$ that can determine whether an observed cumulative distribution $\boldsymbol{G}_{i}^{k}\left(\hat{\boldsymbol{\theta}}, \boldsymbol{\theta}_{p}\right)$ is separated sufficiently from all but one of the training sets with a probability $P_{\text {clas }}$.

\section{ACKNOWLEDGMENT}

The authors would like to thank the Research Council of Norway (RCN) for financial support through projects 203471 CRI SAMCoT and $223254 \mathrm{CoE}$ AMOS and the Swedish Polar Research Secretariat for support through the SWEDARCTIC 2016 research program.

\section{REFERENCES}

[1] N. Bock, "Sustainable Development Considerations in the Arctic," in Environmental Security in the Arctic Ocean, P. A. Berkman and A. N. Vylegzhanin, Eds. Cambridge: Cambridge University Press, 2010, pp. 37-59. [Online]. Available: http://ebooks.cambridge.org/ref/id/CBO9781107415324A009

[2] J. M. Hamilton, "The Challenges of Deep Water Arctic Development," Isope, vol. 8, no. 4, pp. 1-7, 2011.

[3] R. Skjetne, L. Imsland, and S. Løset, "The Arctic DP research project: Effective stationkeeping in ice," Modeling, Identification and Control, vol. 35, no. 4, pp. 191-210, 2014.

[4] K. Eik, "Review of Experiences within Ice and Iceberg Management," Journal of Navigation, vol. 61, no. 04, p. 557, 2008.

[5] H. Bjørklund, A. Sinitsyn, and A. Prusakov, "360 degree camera system for monitoring ice conditions," Proceedings of the 23rd International Conference on Port and Ocean Engineering under Arctic Conditions, 2015.

[6] M. Suominen, J. Kulovesi, M. Lensu, J. Lehtiranta, and P. Kujala, "A Comparison of Shipborne Methods for Ice Thickness Determination," in 22nd international Symposium on Ice, Singapore, 2014.

[7] Q. Zhang and R. Skjetne, Sea Ice Image Processing with MATLAB. Boca Raton: CRC Press, 2018.

[8] H.-M. Heyn, M. Knoche, Q. Zhang, and R. Skjetne, "A system for automated vision-based sea-ice concentration detection and floe-size distribution indication from an icebreaker," in Proceedings of the 36th International Conference on Ocean, Offshore \& Arctic Engineering, Trondheim, 2017.

[9] Q. Zhang and R. Skjetne, "Image Processing for Identification of Sea-Ice Floes and the Floe Size Distributions," IEEE Transactions on Geoscience and Remote Sensing, vol. 53, no. 5, pp. 2913-2924, 52015. 
[10] - "Image Techniques for Identifying Sea-Ice Parameters," Modeling, Identification and Control: A Norwegian Research Bulletin, vol. 35, no. 4, pp. 293-301, 2014.

[11] P. Lu and Z. Li, "A method of obtaining ice concentration and floe size from shipboard oblique sea ice images," IEEE Transactions on Geoscience and Remote Sensing, vol. 48, no. 7, pp. 2771-2780, 2010

[12] W. Lu, Q. Zhang, R. Lubbad, S. Løset, and R. Skjetne, "A Shipborne Measurement System to Acquire Sea Ice Thickness and Concentration at Engineering Scale Installation of Cameras," in Arctic Technology Conference 2016, 2016.

[13] Ø. K. Kjerstad, S. Løset, R. Skjetne, and R. A. Skarbø, "An Ice-Drift Estimation Algorithm Using Radar and Ship Motion Measurements," IEEE Transactions on Geoscience and Remote Sensing, pp. 1-13, 2018.

[14] A. Parsa and D. Smith, "Remote Sensing of Sea Ice Using Co- and Cross-Polarization Measurements with Shipborne Radar," International Journal of Offshore and Polar Engineering, vol. 26, no. 4, pp. 321-326, 2016.

[15] T. Toyota, K. Nakamura, S. Uto, K. I. Ohshima, and N. Ebuchi, "Retrieval of sea ice thickness distribution in the seasonal ice zone from airborne L-band SAR," International Journal of Remote Sensing, vol. 30, no. 12, pp. 3171-3189, 2009.

[16] M. Johnston, R. Frederking, G. Timco, and M. Miles, "Using Motan To Measure Global Accelerations of the Ccgs Terry Fox During Bergy Bit Trials," International Conference on Offshore Mechanics and Arctic Engineering, pp. 1-8, 2004.

[17] O. K. Kjerstad and R. Skjetne, "Disturbance Rejection by Acceleration Feedforward for Marine Surface Vessels," IEEE Access, vol. 4, pp 2656-2669, 2016.

[18] Ø. K. Kjerstad, W. Lu, R. Skjetne, and S. Løset, "A method for realtime estimation of full-scale global ice loads on floating structures," Cold Regions Science and Technology, 2018.

[19] M. Suominen and P. Kujala, "Variation in short-term ice-induced load amplitudes on a ship's hull and related probability distributions," Cold Regions Science and Technology, vol. 106-107, pp. 131-140, 102014.

[20] B. Leira, L. Børsheim, . Espeland, and J. Amdahl, "Ice-load estimation for a ship hull based on continuous response monitoring," Proceedings of the Institution of Mechanical Engineers, Part M: Journal of Engineering for the Maritime Environment, vol. 223, no. 4, pp. 529-540, 112009.

[21] M. Johnston, R. Frederking, G. Timco, and M. Miles, "MOTAN: A Novel Approach for determining Ice-Induced Global Loads on Ships," Proceedings MARI-TECH 2003, no. 05, pp. 1-17, 2003.

[22] S. van der Werff, A. Haase, R. Huijsmans, and Q. Zhang, "Influence of the ice concentration on the ice loads on the hull of a ship in a managed ice field," in Proceedings of the ASME 2012 31st International Conference on Ocean, Offshore and Arctic Engineering, Rio de Janeiro, 2012, pp. 1-7.

[23] S. Løset, K. N. Shkhinek, O. T. Gudmestad, and K. V. Høyland, Actions from ice on Arctic offshore and coastal structures. LAN, 2006.

[24] K. Riska, "Ship-ice interaction in ship design: Theory and practice," Developed under the Auspices of the UNESCO, Tech. Rep., 2011.

[25] I. J. Jordaan, "Mechanics of ice - structure interaction," Engineering Fracture Mechanics, vol. 68, pp. 1923-1960, 2001.

[26] A. Suyuthi, B. J. Leira, and K. Riska, "Short term extreme statistics of local ice loads on ship hulls," Cold Regions Science and Technology, vol. 82, pp. 130-143, 2012.

[27] _ _A generalized probabilistic model of ice load peaks on ship hulls in broken-ice fields," Cold Regions Science and Technology, vol. 97, pp. 7-20, 2014.

[28] H.-M. Heyn, G. Udjus, and R. Skjetne, "Distributed motion sensing on ships," in Oceans 17 Conference, 2017.

[29] P. Kujala, On the statistics of ice loads on ship hull in the Baltic /. Finish Academy of Technology, 1994.

[30] K. Gårdfeldt and s. Lindgren, "SWEDARCTIC Arctic Ocean 2016," Swedish Polar Research Secretariat, Stockholm, Tech. Rep., 2017.
[31] R. Lubbad, E. V. Raaij, and K. J. Eik, "Oden Arctic Technology Research Cruise 2012," in 22nd International Conference on Port and Ocean Engineering under Arctic Conditions, vol. 2012. Offshore Technology Conference, 10 2013, pp. 1-13. [Online]. Available: http://www.onepetro.org/doi/10.4043/27340-MS

[32] Analog Devices Inc., "Data Sheet ADIS 16364," Analog Devices Inc., Norwood, Tech. Rep., 2011.

[33] T. I. Fossen, Handbook of Marine Craft Hydrodynamics and Motion Control. Chichester, UK: John Wiley \& Sons, Ltd, 42011.

[34] A. Noureldin, T. B. Karamat, and J. Georgy, Fundamentals of inertial navigation, satellite-based positioning and their integration. Berlin, Heidelberg: Springer Berlin Heidelberg, 2013.

[35] T. H. Bryne, J. M. Hansen, R. H. Rogne, N. Sokolova, T. I. Fossen, and T. A. Johansen, "Nonlinear Observers for Integrated INS $\backslash$ GNSS Navigation: Implementation Aspects," IEEE Control Systems, vol. 37, no. 3, pp. 59-86, jun 2017.

[36] J. Matusiak, "Dynamic loads and response of icebreaker Sisu during continuous icebreaking," Winter Navigation research board, Helsinki, Tech. Rep., 1982.

[37] H.-M. Heyn and R. Skjetne, "Time-frequency analysis of acceleration data from ship-ice interaction events," Cold Regions Science and Technology, no. January 2017, pp. 1-14, feb 2018.

[38] M. Blanke, M. Kinnaert, J. Lunze, and M. Staroswiecki, Diagnosis and Fault-Tolerant Control, 3rd ed. Berlin, Heidelberg: Springer Berlin Heidelberg, 2016.

[39] H. Joutsijoki and M. Juhola, "Comparing the One-vs-One and Onevs-All Methods in Benthic Macroinvertebrate Image Classification," in Machine Learning and Data Mining in Pattern Recognition, P. Perner, Ed. Berlin, Heidelberg: Springer Berlin Heidelberg, 2011, pp. 399413.

[40] S. M. Kay, Fundamentals of statistical signal processing, Vol. II: Detection Theory. Signal Processing. Upper Saddle River, NJ: Prentice Hall, 1998.

[41] R. Domingues, M. Filippone, P. Michiardi, and J. Zouaoui, "A comparative evaluation of outlier detection algorithms: Experiments and analyses," Pattern Recognition, vol. 74, pp. 406-421, 2018.

[42] S. Nadarajah and S. Kotz, "Mathematical Properties of the Multivariate t Distribution," Acta Applicandae Mathematicae, vol. 89, no. 1-3, pp. 53-84, 2005.

[43] S. Kotz and S. Nadarajah, Multivariate $t$ Distributions and Their Applications. Cambridge: Cambridge University Press, 2004.

[44] D. Peel and G. J. McLachlan, "Robust mixture modeling using the $t$ distribution," Journal Statistics and Computing, vol. 10, pp. 339-348, 2000.

[45] C. Aeschliman, J. Park, and A. C. Kak, "A novel parameter estimation algorithm for the multivariate t-distribution and its application to computer vision," in European Conference on Computer Vision. Springer, 2010, pp. 594-607.

[46] Z. Khan, T. Balch, and F. Dellaert, "MCMC-based particle filtering for tracking a variable number of interacting targets," IEEE Transactions on Pattern Analysis and Machine Intelligence, vol. 27, no. 11, pp. 18051819, 2005.

[47] A. Willersrud, M. Blanke, L. Imsland, and A. Pavlov, "Drillstring Washout Diagnosis Using Friction Estimation and Statistical Change Detection," IEEE Transactions on Control Systems Technology, vol. 23, no. 5, pp. 1886-1900, 2015.

[48] M. Ghane, A. Nejad, M. Blanke, Z. Gao, and T. Moan, "Statistical fault diagnosis of wind turbine drivetrain applied to a 5MW floating wind turbine," Journal of Physics: Conference Series, vol. 753, no. 5, 2016.

[49] S. Nadarajah and S. Kotz, "Estimation methods for the multivariate t distribution," Acta Applicandae Mathematicae, vol. 102, no. 1, pp. 99-118, 2008.

[50] C. Liu and D. B. Rubin, "ML Estimation of the t Distribution using EM and its extensions, ECM and ECME," Statistica Sinica, vol. 5, no. 1, pp. 19-39, 1995. 
[51] J. M. Joyce, "Kullback-Leibler Divergence," in International Encyclopedia of Statistical Science. Berlin, Heidelberg: Springer Berlin Heidelberg, 2011, pp. 720-722.

[52] J. Beirlant, E. Dudewicz, L. Györfi, and E. Van der Meulen, "Nonparametric entropy estimation: An overview," International Journal of Mathematical and Statistical Sciences, vol. 6, no. 1, pp. 17-39, 1997.

[53] J.-L. Guerrero-Cusumano, "A measure of total variability for the multivariate $\mathrm{t}$ distribution with applications to finance," Information Sciences, vol. 92, no. 1, pp. 47-63, 1996.

[54] C. Sammut, "Concept Learning," in Encyclopedia of Machine Learning and Data Mining, 2017, pp. 256-259.

[55] J. Furnkranz, "Decision Tree," in Encyclopedia of Machine Learning and Data Mining, 2017, pp. 256-259.

[56] S. K. Murthy, "Automatic Construction of Decision Trees from Data: A Multi-Disciplinary Survey," Data Mining and Knowledge Discovery, vol. 2, no. 4, pp. 345-389, 1998.

[57] X. Zhang, "Support Vector Machine," in Encyclopedia of Machine Learning and Data Mining, 2017, pp. 1214-1220.

[58] Ø. Kjerstad, I. Metrikin, S. Løset, and R. Skjetne, "Experimental and phenomenological investigation of dynamic positioning in managed ice," Cold Regions Science and Technology, vol. 111, pp. 67 - 79, 2015.

[59] U. Nielsen, R. Galeazzi, and A. H. Brodtkorb, "Evaluation of shipboard wave estimation techniques through model-scale experiments," in Proceedings of OCEANS'16, 2016.

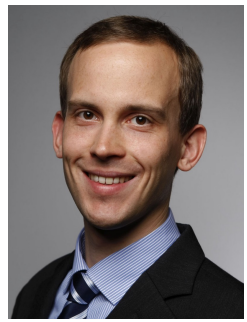

Hans-Martin Heyn received the M.Sc in electrical engineering from RWTH Aachen University, Germany in 2013. In 2013 and 2014 he worked with projects on electromobility and battery charging algorithms at the E.ON Energy Research Center in Aachen, Germany. Since 2014 he pursuits a PhD at the Centre for Autonomous Marine Operations and Systems (AMOS) at the Department of Marine Technology at the Norwegian University of Science and Technology in Trondheim, Norway. His research interests are within autonomous robots and systems, distributed sensor systems, statistical signal processing, and fault diagnosis.

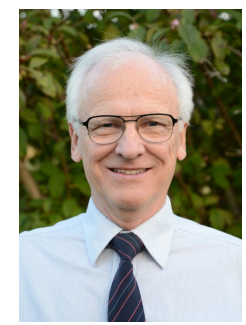

Mogens Blanke received the M.Sc. and Ph.D. degrees in electrical engineering from the Technical University of Denmark (DTU), Lyngby, Denmark, in 1974 and 1982, respectively. He has been Professor in Automation and Control at DTU since 2000 and, since 2005, also Adjunct Professor at the Center for Excellence fo Autonomous Marine Operations and Systems (AMOS) at the Norwegian University of Science and Technology, Trondheim, Norway. He was formerly full professor at Aalborg University (1990-1999), Head of Marine Division at Lyngs $\varnothing$ Marine, Denmark (1985-1990), and was systems analyst at the European Space agency (1975-76). He has authored more than 270 scientific papers, book chapters and books including a widely recognized textbook on Diagnosis and Fault-tolerant Control. His research interests include general subjects in automation and control, fault diagnosis and prognosis, and fault-tolerant control systems. Mogens Blanke was technical editor for IEEE Transactions of Aerospace and Electronic Systems during a decade and has been dedicated editor for several special issues of international journals. He is currently associate editor for Control Engineering Practice. He received several awards and recognitions, including the ASME DSCD Rudolf Kalman Best Paper Award in 2018.

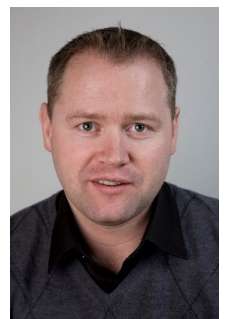

Roger Skjetne received his MSc degree in 2000 from the University of California at Santa Barbara (UCSB) and his $\mathrm{PhD}$ degree in 2005 from the Norwegian University of Science and Technology (NTNU) on control engineering. He holds an Exxon Mobil prize for his PhD thesis at NTNU. Prior to his studies, he worked as a certified electrician for Aker Elektro AS on numerous oil installations for the North Sea, and in 2004-2009 he was employed in Marine Cybernetics AS, working on HardwareIn-the-Loop simulation for testing marine control systems. From August 2009 he has held the Kongsberg Maritime chair of Professor in Marine Control Engineering at the Department of Marine Technology at NTNU. His research interests are within dynamic positioning of marine vessels, Arctic stationkeeping and Ice Management systems, control of shipboard hybrid electric power systems, nonlinear motion control of marine vehicles, and autonomous ships and marine robots. 